\title{
TRABES ACARTELADAS DE CONCRETO REFORZADO CONTINUAS DISEÑADAS PARA FALLAR POR CORTANTE. PARTE 2: MECANISMO DE RESISTENCIA A CORTANTE
}

\author{
Arturo Tena Colunga ${ }^{(1)}$, Luis Angel Urbina Californias ${ }^{(2)}$ y Hans I. Archundia Aranda ${ }^{(3)}$ \\ RESUMEN
}

Se presentan resultados e interpretaciones de un programa experimental donde se han ensayando trabes acarteladas de concreto reforzado en condiciones de continuidad y sujetas a cargas cíclicas reversibles que permitan estudiar su comportamiento a cortante. Se estudiaron cuatro trabes acarteladas y una prismática de concreto reforzado que fueron diseñadas para fallar a cortante cuando disponen de refuerzo por cortante en la zona de las cartelas. Todos los especímenes tienen una longitud de acartelamiento de un tercio del claro libre y cubren uniformemente ángulos de acartelamiento entre cero (prismática) y diez grados. Los especímenes fueron ensayados a través de un patrón de cargas cíclicas reversibles y crecientes controladas por desplazamiento, donde se sometieron a dos ciclos completos a la misma amplitud de desplazamiento, aumentando la amplitud de los ciclos de desplazamientos conforme a un patrón geométrico establecido. Las variables en estudio con respecto al mecanismo resistente a cortante son: (a) el ángulo de acartelamiento y su impacto en la resistencia del concreto, (b) la contribución del acero de refuerzo longitudinal inclinado, (c) la contribución del refuerzo transversal y, d) el ángulo de la grieta principal por cortante. Además, se examinaron las diferencias de comportamiento que presentan las trabes acarteladas con respecto a las prismáticas y se evalúan ecuaciones de diseño propuestas basadas en: a) el método de las secciones $\mathrm{y}, \mathrm{b}$ ) modelos de puntales y tensores (modelos de armadura). Se comprueba que con las ecuaciones propuestas se estiman valores de resistencia a cortante razonables para fines de diseño.

Palabras clave: trabes acarteladas; resistencia a cortante; método de las secciones; puntales y tensores

\section{CONTINUOUS REINFORCED CONCRETE HAUNCHED BEAMS DESIGNED TO FAIL IN SHEAR. PART 2: SHEAR-RESISTING MECHANICS}

\begin{abstract}
Research results and interpretations of the testing of five prototype continuous reinforced concrete beams (four haunched and one prismatic) designed to develop a shear failure under cyclic loading are presented. Subject beams were tested with minimum shear reinforcement. The studied haunched

Artículo recibido el 29 de julio de 2016 y aprobado para su publicación el 2 de octubre de 2017. Se aceptarán comentarios y/o discusiones hasta cinco meses después de su publicación.

(1) Profesor, Departamento de Materiales, Universidad Autónoma Metropolitana Azcapotzalco, Av. San Pablo 180, Col. Reynosa Tamaulipas, 02200 México, DF, e-mail: atc@ correo.azc.uam.mx

(2) Profesor, Universidad Politécnica de Chimalhuacán, Emiliano Zapata S/N, Col. Transportistas, 56363 Chimalhuacán, Edo. México, e-mail: ucla_1985@yahoo.com

(3) Profesor Departamento de Materiales, Universidad Autónoma Metropolitana Azcapotzalco, Av. San Pablo 180, Col. Reynosa Tamaulipas, 02200 México, DF, e-mail: $\underline{\text { archundia@azc.uam.mx }}$
\end{abstract}


length is one-third the effective span of the beam. The considered angles of slope of haunch from horizontal vary from $0^{0}$ (prismatic) to $10^{\circ}$. Cyclic tests were displacement-controlled, and two cycles at the same displacement were set in the displacement history which considers a geometrical increment of target displacements. The parameters under study with respect to the shear-resisting mechanism are: (a) the angle of haunch from horizontal and its impact on the concrete strength, (b) the contribution of the inclined longitudinal steel reinforcement, (c) the contribution of the transverse steel reinforcement and, d) the angle of inclination of the main shear crack. Previously proposed design equations were examined based upon: a) the section approach and, b) strut-and-tie (truss model). It was confirmed that, for design purposes, reasonable assessments of the shear strength of haunched beams are obtained with the proposed equations.

Keywords: haunched beams; shear strength; section design approach; strut-and-tie models

\section{ANTECEDENTES EXPERIMENTALES}

El uso de trabes acarteladas en edificios y puentes en el mundo es frecuente, sobre todo en México, como se ha discutido e ilustrado en el artículo compañero (Tena et al. 2017). Sin embargo, son muy reducidas las investigaciones experimentales sobre el comportamiento de trabes acarteladas de concreto reforzado (TACR), y prácticamente todas se han limitado a estudiar su mecanismo resistente a cortante.

En investigaciones experimentales previamente hechas por este equipo de investigación, se formularon recomendaciones para el diseño por cortante de las TACR con base en el análisis y revisión crítica de la información experimental reportada con anterioridad por otros investigadores (Mörsch 1952, Debaiky y El-Niema 1982, Stefanou 1983, El-Niema 1988 y MacLeod y Houmsi 1994) y, principalmente, el ensaye de diez trabes simplemente apoyadas cargadas monótonamente (Archundia 2004, Archundia et al. 2005 y 2006, Tena-Colunga et al. 2008) y otras 10 trabes gemelas cargas cíclicamente (Grande 2005 y 2009, Tena-Colunga et al. 2007, Archundia-Aranda y Tena-Colunga 2008, Archundia-Aranda et al. 2013, Archundia 2013, Archundia y Tena 2015a). Los ángulos de acartelamiento estudiados fueron $0^{\circ}, 3^{\circ}, 6^{\circ}, 9^{\circ}$ y $12^{\circ}$. Estos ángulos cubren perfectamente la práctica observada en la ciudad de México, donde se encuentran trabes acarteladas con ángulos entre cinco y ocho grados.

Las aportaciones más importantes de estas investigaciones previas se reportan en Archundia (2004), Archundia et al. (2005 y 2007), Tena-Colunga et al. (2008), Grande (2009), Archundia-Aranda et al. (2013), Archundia (2013) y Archundia y Tena (2015a). Entre otras cosas, se propone una ecuación general basada en el método de las secciones para determinar la resistencia a cortante de trabes acarteladas de concreto reforzado que es bastante robusta (Tena-Colunga et al. 2008, Archundia-Aranda et al. 2013, Tena et al. 2017), pero que al parecer pudiera ser demasiado conservadora para trabes acarteladas en una condición de continuidad, lo que antes de la investigación experimental en curso no se podía asegurar, dado que los únicos datos experimentales disponibles para comparación eran los del trabajo de MacLeod y Houmsi (1994), donde las trabes acarteladas no tienen refuerzo por cortante.

El trabajo posteriormente publicado por Pérez-Caldentey et al. (2012) en trabes sin refuerzo por cortante en condiciones de continuidad no proporciona mucha información que sea útil para este propósito, dado que se concentró en evaluar el impacto del tipo de carga monótonamente creciente aplicada (puntual, uniformemente distribuida y triangularmente distribuida). El trabajo de referencia sólo reporta los valores últimos obtenidos en sus experimentos, no proporcionando detalles de los resultados experimentales (por ejemplo, progresión de patrones de agrietamiento, ubicación de la instrumentación externa e interna, curvas globales carga-deformación, resultados de la instrumentación interna, en caso de 
existir, etc.). Cabe señalar que las dimensiones de los especímenes son importantes (sección transversal máxima de $25 \mathrm{~cm}$ x $60 \mathrm{~cm}$ y longitudes del claro del voladizo de $3.195 \mathrm{~m}$ y $4.845 \mathrm{~m}$ ).

En Archundia (2013) se propone toda una metodología basada en modelos de armadura para determinar la resistencia a cortante, incluyendo ecuaciones de diseño basadas en el comportamiento mecánico, donde se calcula la contribución del acero y del concreto sin el uso de ajustes estadísticos de datos experimentales, y que se presenta de una manera más sintética en Archundia y Tena (2015a).

Por ello, este grupo de investigación continúa este esfuerzo, desarrollando un programa experimental donde se están ensayando trabes acarteladas de concreto reforzado en condiciones de continuidad y sujetas a cargas cíclicas reversibles, que permitan estudiar su comportamiento a cortante, para poder cuantificar con base experimental los parámetros más significativos que afectan su comportamiento. En las siguientes secciones se discute con detalle el mecanismo resistente a cortante, evaluado experimentalmente, y la validez de las ecuaciones que se han propuesto para su diseño, derivadas tanto del método del equilibrio en la sección crítica, como del método de puntales y tensores.

\section{ENSAYES EXPERIMENTALES}

Como se describe con detalle en el artículo compañero (Tena et al. 2017), los especímenes de prueba se diseñaron para fallar a cortante utilizando una adaptación de la fórmula propuesta para trabes acarteladas simplemente apoyadas por el método de las secciones (Tena-Colunga et al. 2008), y se construyeron en el Laboratorio de Modelos Estructurales Intermedios de la UAM-A, con el apoyo de numerosos alumnos de la Licenciatura en Ingeniería Civil.

Los detalles del diseño, los armados obtenidos, la geometría de los especímenes y su nomenclatura se describen y presentan en el artículo compañero (Tena et al. 2017) y en Urbina (2013), y se omiten aquí en aras de salvar espacio y discutir con mayor detalle las interpretaciones de los resultados obtenidos experimentalmente. Sin embargo, se considera pertinente mostrar nuevamente la instrumentación típica externa de los especímenes en estudio con base en transductores de desplazamiento (TI- $i$ y TD- $i$ ), micrómetros (TRI y TRD), deformímetros de concreto ( $\mathrm{SGC} i$ ) y celdas de carga (figura 1), con la finalidad de facilitar las interpretaciones hechas a partir de las lecturas registradas en ellos.
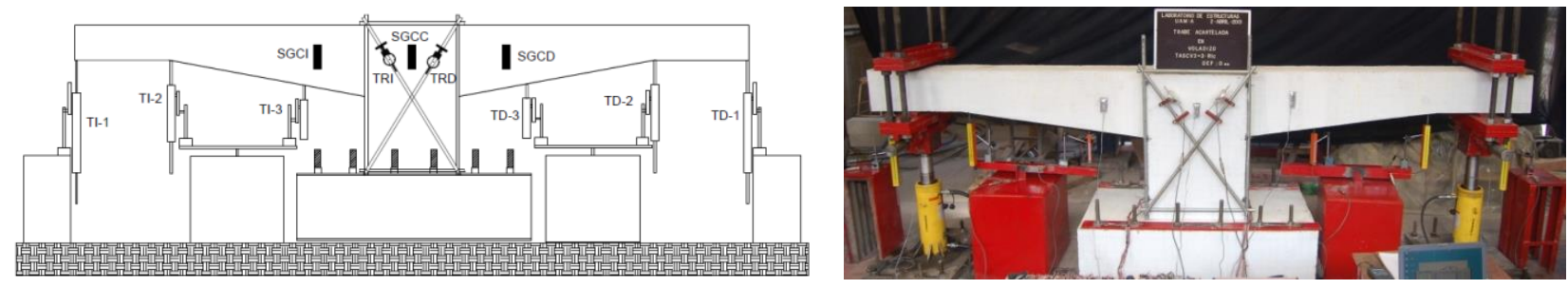

Figura 1. Instrumentación externa del espécimen TASCV3 $\alpha 3-R 1 c$

Los patrones de desplazamientos se aplicaron cíclicamente por medio de gatos hidráulicos de doble acción (figura 1) con capacidad de 95 toneladas conforme al esquema indicado en la figura 2 y se describe con detalle en el artículo compañero (Tena et al. 2017). Como también se explica en el artículo compañero, los ensayes se controlaron por desplazamientos medidos en el vértice (TI-2 y TD-2, figura 1). Para cada sentido de carga (-P y $+\mathrm{P}$, figura 2) y cada medio ciclo de carga, se realizó una repetición para evaluar de esa forma la degradación de la rigidez y resistencia del espécimen para un mismo desplazamiento. En la figura 3 se muestra esquemáticamente la historia de desplazamientos tipo utilizada en todos los ensayes, donde las deflexiones negativas indican que la carga se aplica hacia abajo (momento negativo y cortante negativo). Los ensayes se terminaron cuando los elementos ya no eran capaces de 
soportar carga debido al daño presentado. Los colores mostrados en cada medio ciclo ilustrado en la figura 3 corresponden a los colores con los que se marcaron físicamente las grietas en esos medios ciclos (por ejemplo, figuras 4 a a 8a).

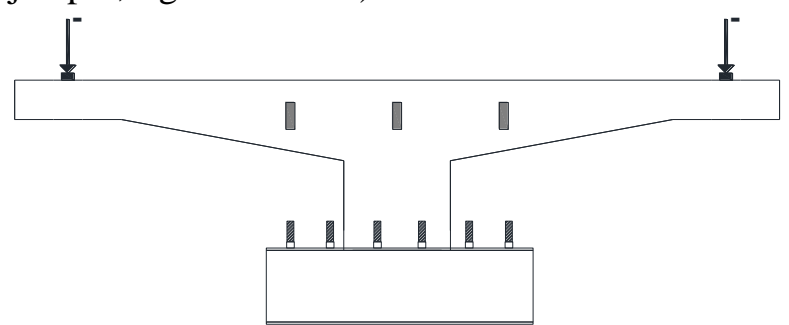

a) Dirección momento negativo

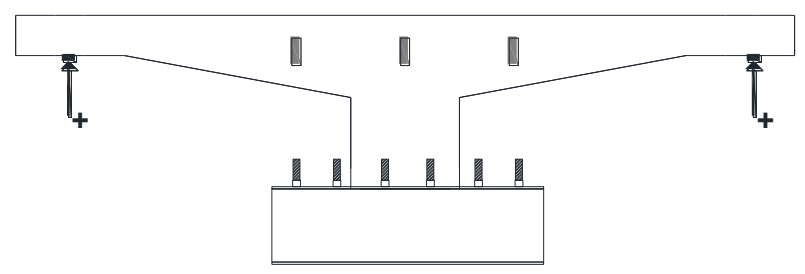

b) Dirección momento positivo

Figura 2. Esquema de la aplicación de la carga cíclica

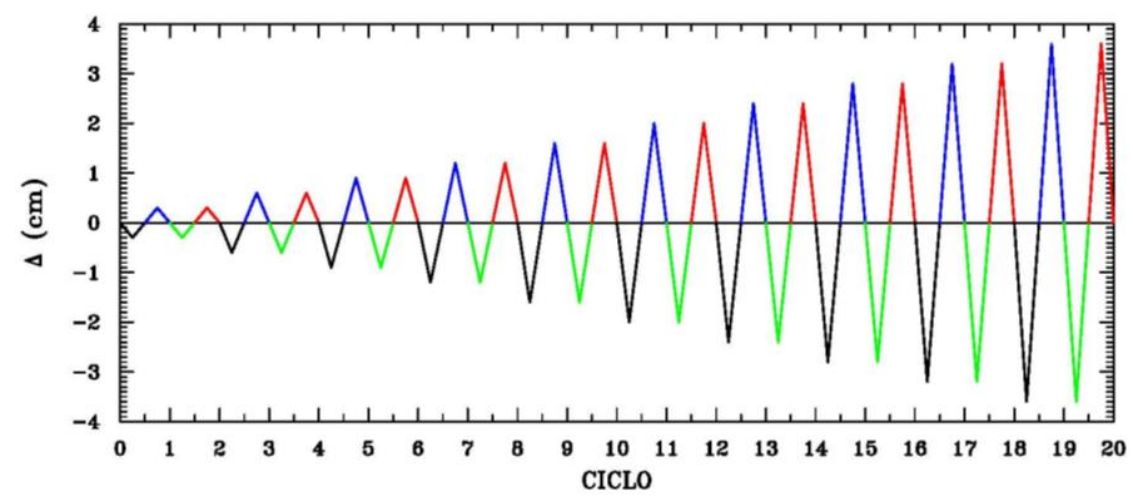

Figura 3. Historia cíclica de desplazamientos aplicada en los especímenes

\section{MECANISMO RESISTENTE A CORTANTE EVALUADO EXPERIMENTALMENTE}

Con base en los datos capturados durante los ensayes experimentales (cargas aplicadas, desplazamientos experimentados, deformaciones en el acero de refuerzo longitudinal y transversal), se pudieron trazar las curvas de histéresis carga-desplazamiento para los distintos puntos del ensaye (Urbina 2013, Aranda 2015, Tena et al. 2017), así como curvas carga-deformación para cada punto monitoreado con los deformímetros (Urbina 2013). También se llevó un registro fotográfico y una medición de anchura de grietas máximas durante el progreso de la prueba para cada desplazamiento objetivo (positivo y negativo), y al final de cada par de ciclos a la misma amplitud, lo que permitió posteriormente trazar esquemas del progreso evolutivo de grietas (por ejemplo, Urbina 2013, Tena et al. 2017).

Como se explica en el artículo compañero (Tena et al. 2017), a partir de los resultados experimentales se obtuvieron las deflexiones y los cortantes asociados al primer agrietamiento diagonal $\left(\Delta_{\text {agr }}\right.$ y $\left.V_{\text {agr }}\right)$, último o de falla $\left(\Delta_{\mathrm{u}}\right.$ y $\left.V_{\mathrm{u}}\right)$ y al colapso $\left(\Delta_{\text {clps }} \mathrm{y} V_{\text {clps }}\right)$. El primer agrietamiento diagonal se define en función de la aparición de la(s) primera(s) grieta(s) inclinada(s). El estado último o de falla se asoció con la aparición de la primera grieta diagonal frágil, súbita e importante que pone en riesgo la estabilidad del espécimen. El estado de colapso se definió cuando se presentaban desconchamientos y desprendimientos de concreto masivos en la zona de la aplicación de la carga, y en la parte superior e inferior a lo largo de la viga, asociado normalmente a una reducción abrupta de la capacidad de carga del espécimen. Estos cortantes y deflexiones características permiten describir el comportamiento de los especímenes. 
Para evaluar el mecanismo resistente a cortante con base experimental, se procedió de la siguiente manera. Para definir el cortante efectivamente aplicado externamente $\left(V_{e f}\right)$, se corrigió el cortante externamente aplicado con los gatos hidráulicos $(V)$ con el cortante debido al peso propio $\left(V_{\mathrm{pp}}\right)$ de la viga en la sección donde se presentó la grieta principal por cortante. Como se puede deducir simplemente de la figura 2, el cortante debido al peso propio se debe sumar al aplicado con los gatos hidráulicos para momento y cortante negativos (figura 2a), pues la carga se aplica en la dirección de la gravedad, mientras que se debe restar en la dirección del momento y cortante positivos (figura 2b), pues la carga se aplica en dirección contraria a la gravedad.

El cortante nominal resistido internamente por las vigas en la sección crítica de interés $\left(V_{n}\right)$, en la condición de continuidad, se determinó como:

$$
\begin{aligned}
& V_{n}=V_{c}+V_{s} \\
& V_{c}=V_{p c}+V_{r i}
\end{aligned}
$$

donde $V_{c}$ es la contribución total del concreto, que toma en cuenta la potencial contribución del refuerzo inclinado y por efecto de dovela, $V_{p c}$ es la contribución nominal del concreto, $V_{r i}$ es la contribución del acero de refuerzo longitudinal inclinado, y $V_{s}$ es la contribución del acero de refuerzo transversal.

Con base en la instrumentación interna, se pudo estimar de manera directa la contribución de los estribos, $V_{s}$, simplemente sumando la fuerzas desarrolladas por cada estribo que cruza la grieta crítica por cortante observada experimentalmente y que se indican esquemáticamente en las figuras $4 \mathrm{~b}$ a $8 \mathrm{~b}$, donde con el color verde se identifican las grietas principales a momento negativo (grietas marcadas en negro y verde en las figuras $4 \mathrm{a}$ a $8 \mathrm{a}$ ) y con el color rojo a las grietas principales a momento positivo (grietas marcadas en azul y rojo en las figuras $4 \mathrm{a}$ a $8 \mathrm{a}$ ). Cabe señalar que las grietas principales por cortante migran de la vecindad del nudo viga-columna en las trabes prismáticas (figura $4 \mathrm{~b}$ ) hacia el interior de las cartelas o a la vecindad del vértice en las trabes acarteladas (figuras $5 \mathrm{~b}$ a $8 \mathrm{~b}$ ). La fuerza desarrollada en cada estribo se calculó con base en las lecturas de los deformímetros (strain gages) y suponiendo un comportamiento elasto-plástico perfecto para el acero de refuerzo, dado que las deformaciones unitarias máximas de los estribos no superaron $2 \varepsilon_{y}$ (Urbina 2013). Se utilizó el esfuerzo de fluencia promedio $f_{y}=$ $4,592 \mathrm{~kg} / \mathrm{cm}^{2}$ obtenido experimentalmente para el acero de los estribos (para estas pruebas y coincide con pruebas anteriores).

Si por equilibrio se iguala el cortante efectivo externamente aplicado con el cortante nominal resistido internamente $\left(V_{n}=V_{e f}\right)$, toda vez que se estima directamente $V_{s}$ como se indicó anteriormente, la contribución total del concreto, $V_{c}$, se obtiene indirectamente a partir de la ecuación 1 como $V_{c}=V_{n}-V_{s}$.

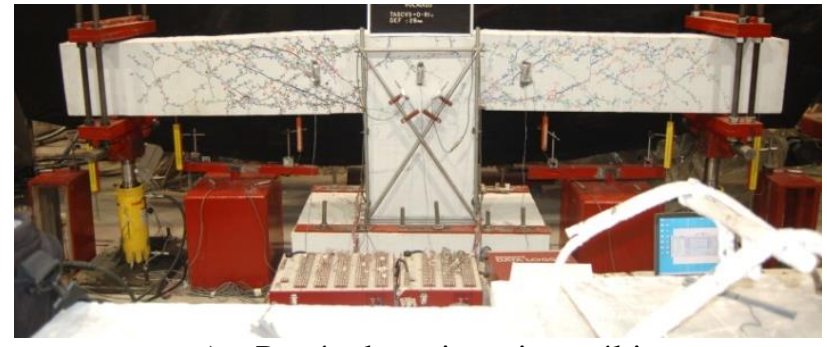

a) Patrón de agrietamiento último

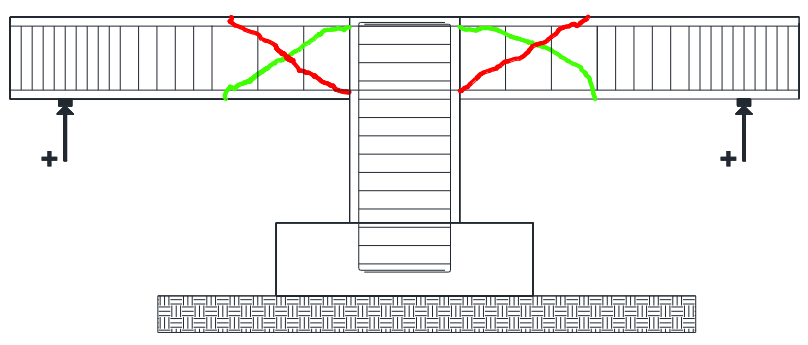

b) Grietas principales a corte

Figura 4. Patrones de agrietamiento, grietas y ángulos principales a cortante observados en las vigas del espécimen TASCV3 $\alpha 0-\mathrm{R} 1 \mathrm{c}$ (prismática) 


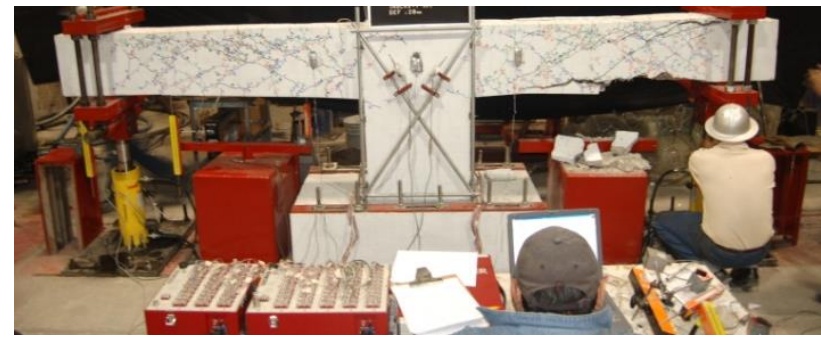

a) Patrón de agrietamiento último

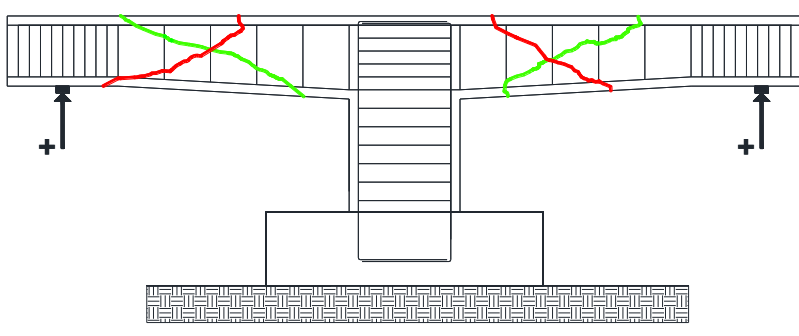

b) Grietas principales a corte

Figura 5. Patrones de agrietamiento, grietas y ángulos principales a cortante observados en las vigas del espécimen TASCV3 $\alpha 1-\mathrm{R} 1 \mathrm{c}\left(\alpha=3.21^{0}\right)$

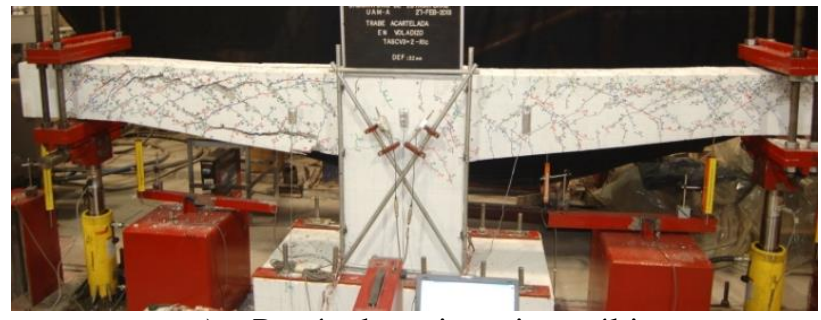

a) Patrón de agrietamiento último

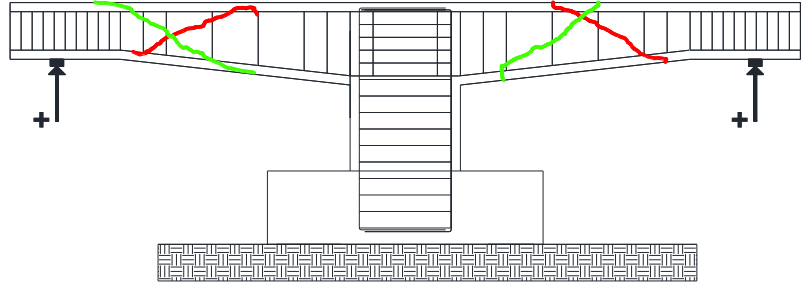

b) Grietas principales a corte

Figura 6. Patrones de agrietamiento, grietas y ángulos principales a cortante observados en las vigas del espécimen TASCV3 $\alpha 2-\mathrm{R} 1 \mathrm{c}\left(\alpha=6.39^{\circ}\right)$

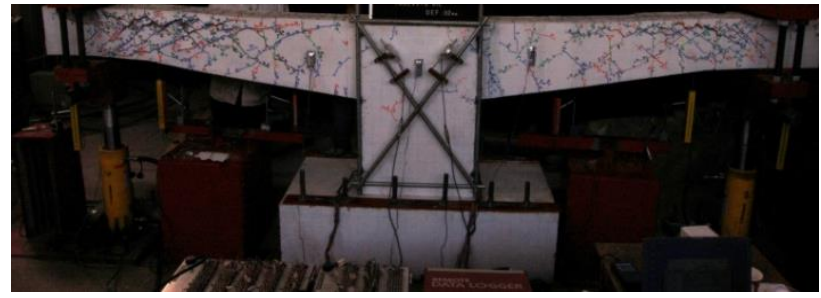

a) Patrón de agrietamiento último

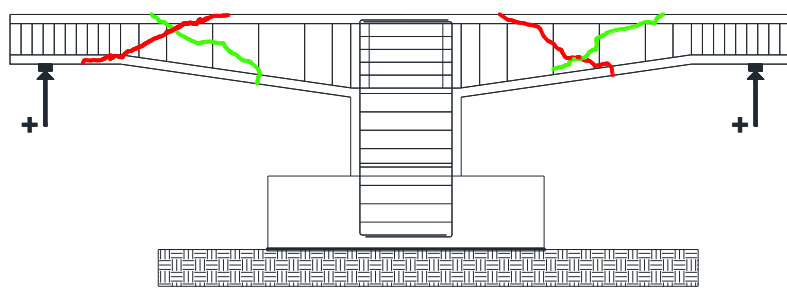

b) Grietas principales a corte

Figura 7. Patrones de agrietamiento, grietas y ángulos principales a cortante observados en las vigas del espécimen TASCV3 $\alpha 3-R 1 c\left(\alpha=8.19^{\circ}\right)$

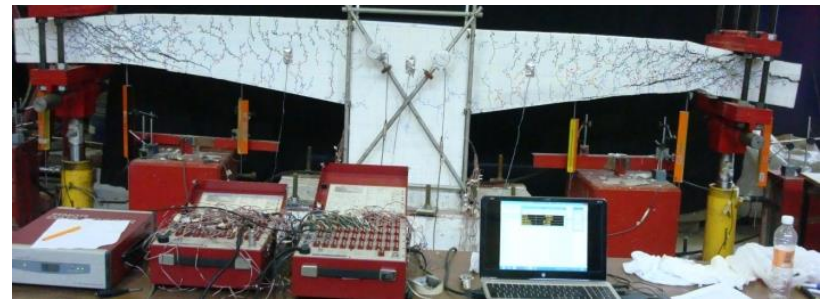

a) Patrón de agrietamiento último

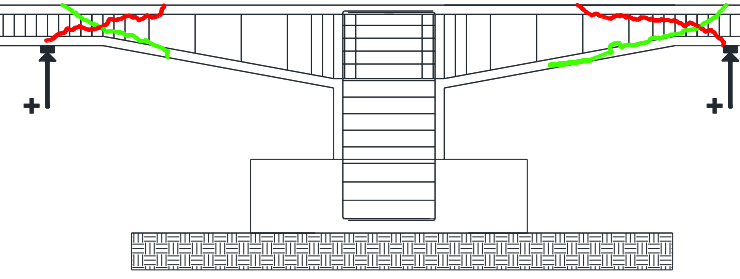

b) Grietas principales a corte

Figura 8. Patrones de agrietamiento, grietas y ángulos principales a cortante observados en las vigas del espécimen TASCV3 $\alpha 4-\mathrm{R} 1 \mathrm{c}\left(\alpha=9.98^{\circ}\right)$

Así, de esta manera, se calcularon los cortantes experimentales $V_{n}, V_{s}$ y $V_{c}$, cuyas envolventes de respuestas máximas para los primeros ciclos de deformación se presentan en la figura 9. Aunque puede resultar obvio, cabe señalar que la estimación más precisa es en el cortante nominal $V_{n}$, le sigue el cortante del refuerzo transversal, $V_{s} \mathrm{y}$, por último $V_{c}$, que se obtiene indirectamente a partir de $V_{n}$ y $V_{s}$. También cabe señalar que por un falso contacto no detectado al inicio de la prueba, se perdieron las lecturas de los strain gages de los estribos del espécimen TASCV $3 \alpha 1-R 1$ c para una distorsión $\Delta \leq 1.0 \%$, y a partir de ahí 
se corrigió el problema y se registraron lecturas, razón por la cual las curvas para $V_{s}$ (figura 9b) están en cero en el siguiente intervalo de distorsiones: $-1.0 \% \leq \Delta \leq 1.0 \%$.

En general, se aprecia en las gráficas de la figura 9 que antes del primer agrietamiento diagonal (distorsiones $\Delta \approx 0.5 \%$ ), la contribución al cortante del acero de refuerzo transversal $\left(V_{s}\right)$ es prácticamente despreciable, pero se incrementa rápidamente a partir de ahí y resiste la mayor parte del cortante nominal, tanto a momento positivo como negativo, para distorsiones mayores al $0.7 \%$ en la trabe prismática (figura 9a), y mayores al 0.9\%-1\% en las trabes acarteladas (por ejemplo, figuras 9c a 9e), lo cual sugiere que la resistencia del concreto se degrada a distorsiones menores en las trabes prismáticas con respecto a las trabes acarteladas. También se aprecia que, en general, a momento positivo, el acero de refuerzo transversal $V_{s}$ resiste prácticamente en su totalidad el cortante aplicado para las cargas máxima y de colapso, mientras que para momento negativo, el concreto resiste aún una porción importante de la carga, principalmente para la carga máxima, pues su contribución a la resistencia al colapso se reduce notablemente.

Con la finalidad de estudiar la contribución al cortante resistente del refuerzo longitudinal inclinado $V_{r i}$ (por ejemplo, ec. 2), ésta se calculó indirectamente a partir de la ecuación 3:

$$
V_{r i}= \pm \frac{M_{c r}}{d_{c r}} \tan \alpha
$$

donde $\alpha$ es el ángulo de acartelamiento, mientras $d_{c r}$ y $M_{c r}$ son respectivamente el peralte efectivo y el momento flexionante desarrollado en la sección crítica donde se presenta la grieta principal por cortante.

Los momentos experimentales $M_{c r}=M_{\text {exp }}$ se calcularon estableciendo el equilibrio de momentos en la sección crítica a cortante (figuras $4 \mathrm{~b}$ a $8 \mathrm{~b}$ ), considerando la curva propuesta por Hognestad (Park y Paulay 1997) para el concreto no confinado, y las lecturas de las deformaciones unitarias del acero de refuerzo longitudinal superior e inferior (inclinado en las trabes acarteladas) en la sección crítica por cortante. Cabe señalar que el equilibrio se estableció: a) proyectando las deformaciones del refuerzo longitudinal inclinado al plano de la falla por cortante, a partir de éstas se calculó la posición del eje neutro y la deformación unitaria máxima del concreto $\left(\varepsilon_{c}\right)$ bajo la hipótesis de la sección plana y, b) en el modelo de Hognestad se emplearon el valor de $\varepsilon_{\mathrm{c}}$ calculado anteriormente y los valores de la resistencia nominal a la compresión $\left(f^{\prime}\right)$ y del módulo de elasticidad $\left(E_{c}\right)$ determinados experimentalmente a partir de las pruebas de cilindros de control probados el día del ensaye, cuyos valores se reportan en la tabla 3 (Urbina 2013), y un esfuerzo de fluencia del acero longitudinal $f_{y}=4,348 \mathrm{~kg} / \mathrm{cm}^{2}$ medido experimentalmente. Por supuesto, bajo este planteamiento de equilibrio existen fuerzas axiales efectivas (compresión y tensión), y este acoplamiento de flexión y carga axial en trabes acarteladas ya ha sido demostrado tanto analíticamente (El-Mezaini et al. 1991) como experimentalmente (Hou et al. 2015). De hecho, antes de la carga máxima, la fuerza neta efectiva calculada fue de compresión y cambió a tensión cercana a esta carga máxima y en su progresión al colapso. También cabe señalar que cuando una grieta principal por cortante se ubicó no cercana a la ubicación de los strain gages que medían las deformaciones del refuerzo longitudinal, o éstos desafortunadamente no registraron lecturas o dejaron de registrar datos al despegarse, las deformaciones se estimaron a partir de interpolaciones lineales de las lecturas de deformímetros fuera de la zona crítica, que afortunadamente fueron los menores de los casos. Como se mencionó anteriormente, $V_{c}$ se obtuvo indirectamente a partir de $V_{n}$ y $V_{s}$. 

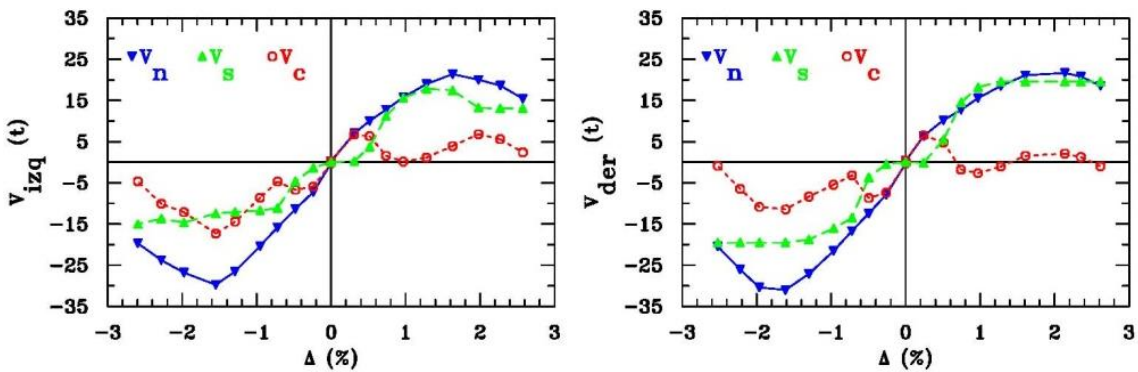

a) TASCV $3 \alpha 0-\mathrm{R} 1 \mathrm{c}$
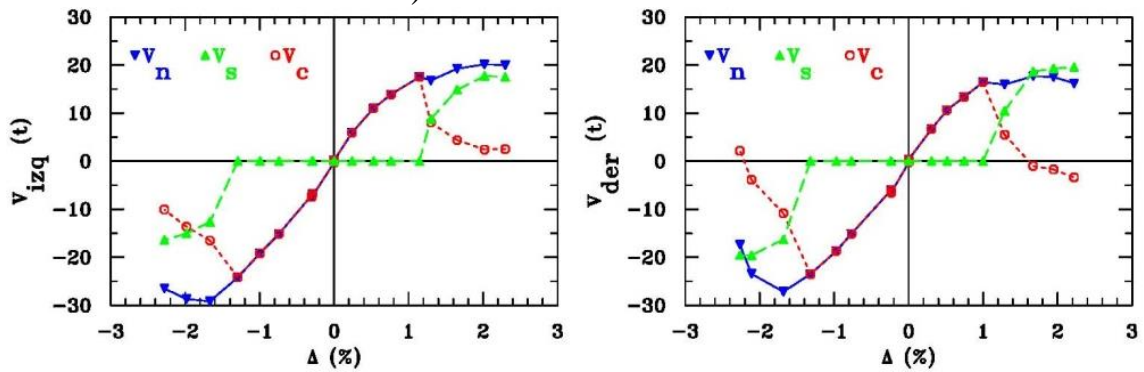

b) TASCV3 $\alpha 1-\mathrm{R} 1 \mathrm{c}$
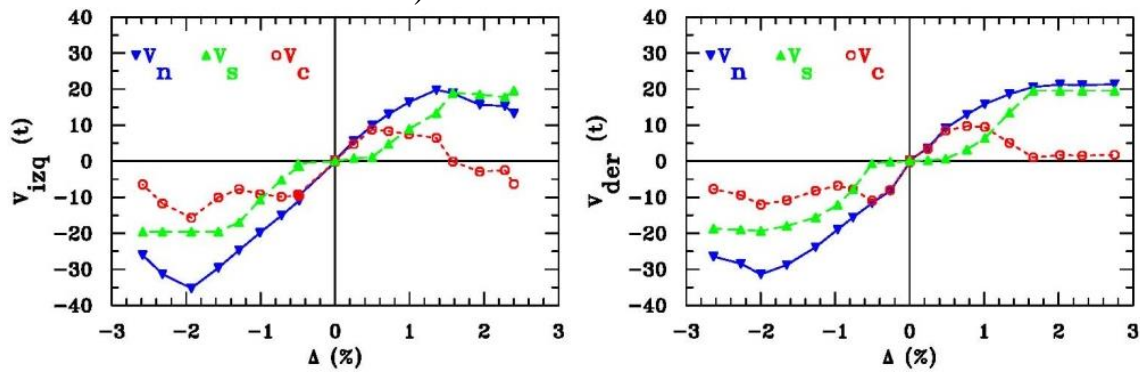

c) TASCV $3 \alpha 2-\mathrm{R} 1 \mathrm{c}$
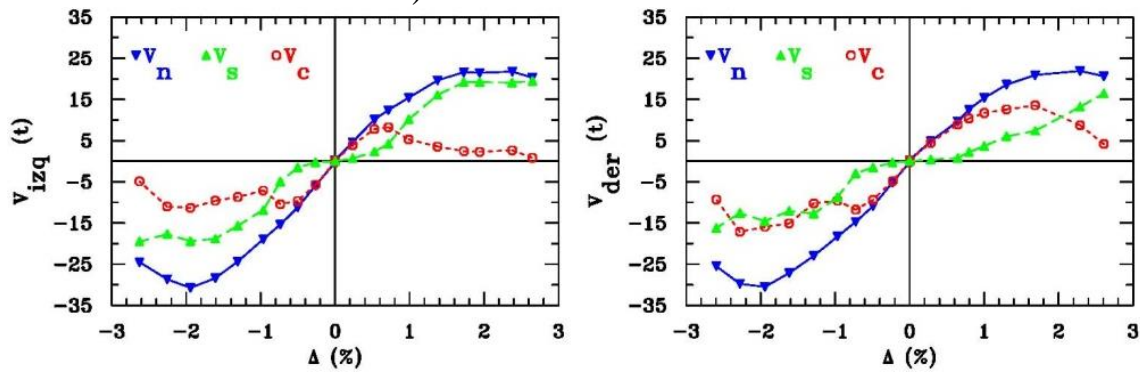

d) TASCV3 $\alpha 3-\mathrm{R} 1 \mathrm{c}$
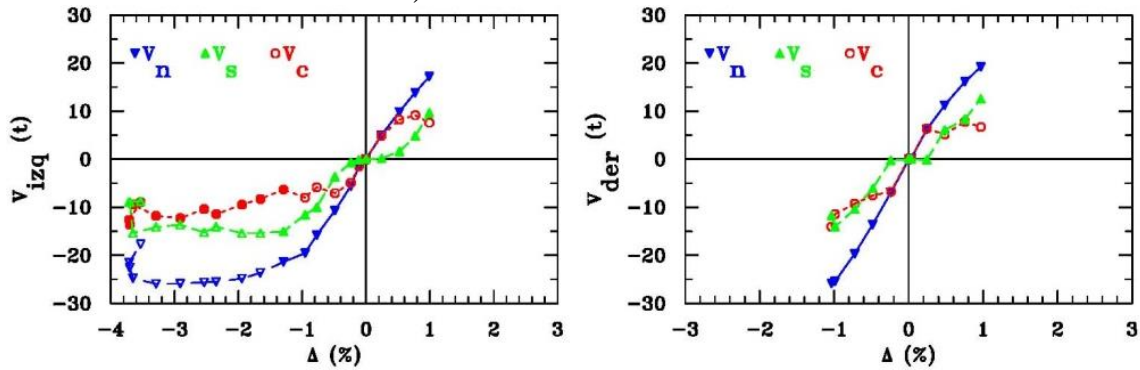

e) TASCV3 $\alpha 4-\mathrm{R} 1 \mathrm{c}$

Figura 9. Envolventes de respuestas máximas (cortantes) de los especímenes en estudio para los primeros ciclos. Cartelas izquierdas y derechas en el lado izquierdo y derecho, respectivamente 
De esta manera, se trazaron las curvas de la contribución al cortante resistente del refuerzo longitudinal inclinado, $V_{r i}$, la cuales se comparan con las de la contribución del concreto $V_{c}$ en la figura 10 para las trabes acarteladas en estudio. Por obvias razones, en la figura 10 no se incluye la trabe prismática. También cabe señalar que para el espécimen TASV3 $\alpha 1-R 1 c$ (figura 10a), la estimación de $V_{c}$ es exagerada en el intervalo de distorsiones $-1.0 \% \leq \Delta \leq 1.0 \%$, dado que no se pudo estimar $V_{s}$ por el problema de falso contacto ya explicado, y eso deberá tomarse en cuenta al evaluar la figura 10a.
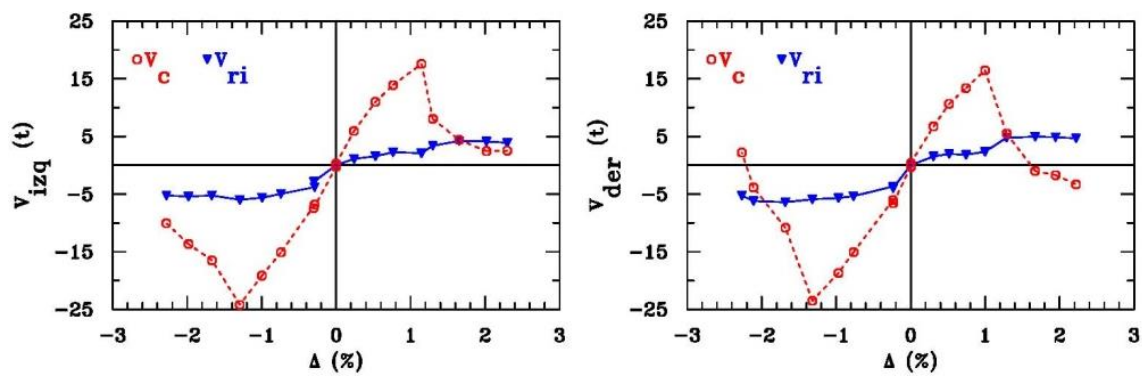

a) TASCV $3 \alpha 1-\mathrm{R} 1 \mathrm{c}$
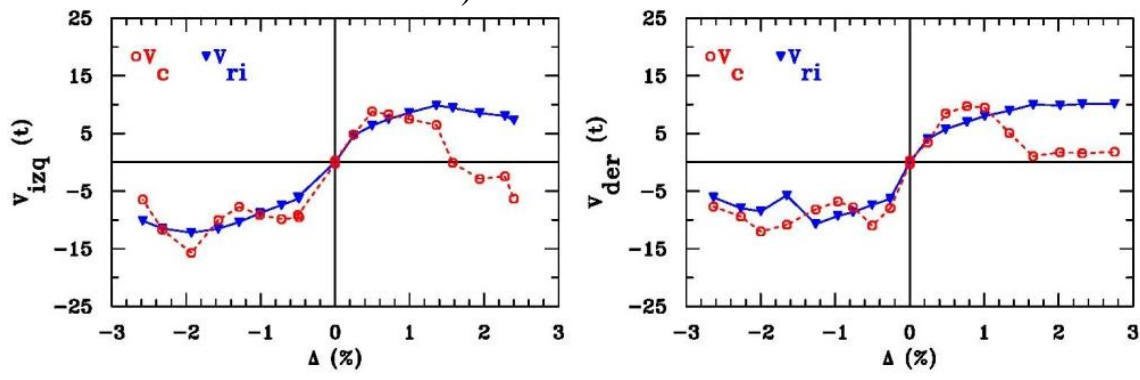

b) TASCV $3 \alpha 2-\mathrm{R} 1 \mathrm{c}$
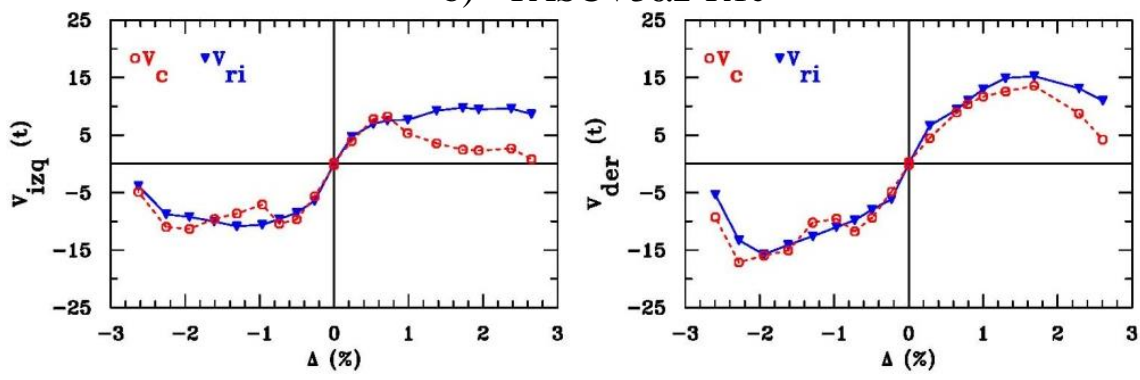

c) TASCV $3 \alpha 3-\mathrm{R} 1 \mathrm{c}$
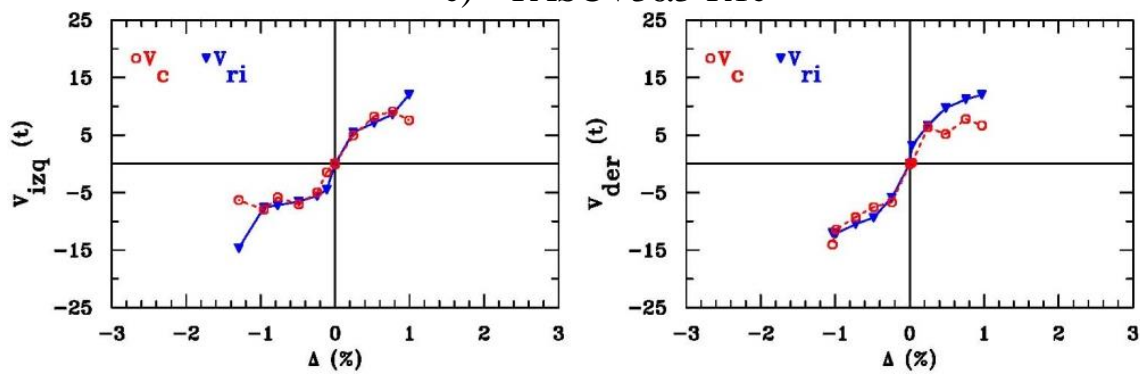

d) TASCV3 $\alpha 4-\mathrm{R} 1 \mathrm{c}$

Figura 10. Envolventes de respuestas máximas de los especímenes en estudio para los primeros ciclos. Cartelas izquierdas y derechas en el lado izquierdo y derecho, respectivamente

Hechas estas precisiones, se aprecia lo siguiente en la figura 10 con respecto a la contribución del refuerzo inclinado a la resistencia al corte: a) su contribución es evidente aún antes del primer 
agrietamiento diagonal para todas las trabes acarteladas en estudio, b) como era de esperarse, su contribución aumenta a medida que el ángulo de acartelamiento aumenta, c) tal vez no tan evidente, a medida que aumenta el ángulo de acartelamiento, se convierte en el mecanismo resistente principal del concreto a cortante, aún a distorsiones pequeñas (figuras 10b, 10c y 10c) y, d) toma prácticamente toda la resistencia a cortante del concreto para distorsiones mayores a $1 \%$, lo que indica que en las cargas máximas y de colapso, el cortante que aporta el refuerzo inclinado en trabes acarteladas es el único que se suma a la contribución de los estribos, pues el concreto en sí agotó prácticamente su resistencia.

En las tablas 1 y 2 se resumen las fuerzas cortantes experimentales resistentes por cada concepto asociados a la carga máxima o última de diseño aplicada, $V_{u}$. Aunque todos los términos involucrados ya fueron explicados, se recuerda que $V_{u e f}$ es el cortante último efectivo, calculado como el cortante externamente aplicado con los gatos hidráulicos $\left(V_{u}\right)$ corregido por el cortante debido al peso propio $\left(V_{p p}\right)$ de la viga en la sección donde se presentó la grieta principal por cortante, según corresponda, y ya se explicó cómo se determinan $V_{s}, V_{c}$ y $V_{r i}$. En las tablas 1 y 2 se indican con asterisco (*) los valores del espécimen TASCV3 $\alpha 4-R 1 c$ que no corresponden a la carga última por el colapso súbito de vértice de la cartela derecha, descrito con mayor detalle en el artículo compañero (Tena et al. 2017), y con rojo los valores de $V_{r i}$ que se obtuvieron a partir de interpolaciones lineales del refuerzo longitudinal fuera de la sección crítica, para el caso que la lectura del strain gage más cercano a dicha sección crítica se perdió poco antes durante la prueba. El valor de $V_{p c}$ se obtuvo indirectamente a partir de la ecuación 2 como $V_{p c}=V_{c}-V_{r i}$ y, en caso de resultar negativo a la carga última, se muestra un guión en rojo.

De las resultados mostrados en la tablas 1 y 2 se confirma lo que se observa en las curvas envolventes, y es que, a excepción del espécimen que falló prematuramente (TASCV3 $\alpha 4-R 1 c)$ por las razones que se explican en el artículo compañero (Tena et al. 2017), las trabes acarteladas resisten un cortante último efectivo similar o aún mayor que las trabes prismáticas de referencias, sobre todo a medida que aumenta el ángulo de acartelamiento, y particularmente a momento negativo. La resistencia a cortante adicional de las trabes acarteladas a un menor volumen de concreto se debe a dos razones: 1) un mayor aporte del refuerzo longitudinal inclinado, tanto a momento positivo y negativo, la cual en general se incrementa a medida que aumenta el ángulo de acartelamiento y, 2) en general, el refuerzo transversal por cortante participa más en la resistencia a medida que aumenta el ángulo de acartelamiento $\alpha$, como consecuencia de que el ángulo de inclinación de la grieta principal por cortante $\theta$ disminuye, como se ilustra más adelante.

Tabla 1. Cortantes últimos medidos en los especímenes en estudio, momento negativo

\begin{tabular}{|c|c|c|c|c|c|c|c|c|c|c|c|c|c|c|}
\hline \multirow{2}{*}{ Espécimen } & \multicolumn{7}{|c|}{ Cartela (viga) izquierda } & \multicolumn{7}{|c|}{ Cartela (viga) derecha } \\
\hline & $\begin{array}{l}\mathbf{V}_{p c} \\
(t)\end{array}$ & $\begin{array}{l}V_{\text {ri }} \\
(t)\end{array}$ & $\begin{array}{l}V_{c} \\
(t)\end{array}$ & $\begin{array}{l}V_{s} \\
(t)\end{array}$ & $\begin{array}{l}V_{u} \\
(t)\end{array}$ & $\begin{array}{l}V_{p p} \\
(t)\end{array}$ & $\begin{array}{l}\mathbf{V}_{\text {uef }} \\
(t)\end{array}$ & $\begin{array}{l}\mathbf{V}_{p c} \\
(t)\end{array}$ & $\begin{array}{l}V_{r i} \\
(t)\end{array}$ & $\begin{array}{l}V_{c} \\
(t)\end{array}$ & $\begin{array}{l}V_{s} \\
(t)\end{array}$ & $\begin{array}{l}V_{u} \\
(t)\end{array}$ & $\begin{array}{l}\mathbf{V}_{p p} \\
(t)\end{array}$ & $\begin{array}{c}V_{\text {uef }} \\
(t)\end{array}$ \\
\hline TASCV3a0-R1c & 17.29 & 0.00 & 17.29 & 12.46 & 29.49 & 0.26 & 29.75 & 11.43 & 0.00 & 11.43 & 19.56 & 30.67 & 0.32 & 30.99 \\
\hline TASCV3a1-R1c & 11.25 & 5.25 & 16.50 & 12.65 & 28.90 & 0.25 & 29.15 & 5.04 & 6.42 & 11.46 & 16.32 & 27.47 & 0.31 & 27.78 \\
\hline TASCV3a2-R1c & 3.49 & 12.21 & 15.71 & 19.56 & 35.06 & 0.21 & 35.27 & 3.52 & 8.52 & 12.05 & 19.33 & 31.05 & 0.32 & 31.38 \\
\hline TASCV3 $\alpha 3-R 1 c$ & 2.09 & 9.23 & 11.33 & 19.41 & 30.49 & 0.25 & 30.74 & 0.17 & 15.78 & 15.95 & 14.54 & 30.25 & 0.25 & 30.49 \\
\hline TASCV3a4-R1c & - & 14.66 & 6.32 & 15.05 & $21.28 *$ & 0.09 & $21.37 *$ & 2.12 & 11.93 & 14.05 & 11.80 & 25.76 & 0.09 & 25.85 \\
\hline
\end{tabular}

Tabla 2. Cortantes últimos medidos en los especímenes en estudio, momento positivo

\begin{tabular}{|c|c|c|c|c|c|c|c|c|c|c|c|c|c|c|}
\hline \multirow[t]{2}{*}{ Espécimen } & \multicolumn{7}{|c|}{ Cartela (viga) izquierda } & \multicolumn{7}{|c|}{ Cartela (viga) derecha } \\
\hline & $\begin{array}{l}V_{p c} \\
(t)\end{array}$ & $\begin{array}{l}V_{\text {ri }} \\
(t)\end{array}$ & $\begin{array}{l}V_{c} \\
(t)\end{array}$ & $\begin{array}{l}V_{s} \\
(t)\end{array}$ & $\begin{array}{l}V_{u} \\
(t)\end{array}$ & $\begin{array}{l}V_{p p} \\
(t)\end{array}$ & $\begin{array}{c}V_{\text {uef }} \\
\text { (t) }\end{array}$ & $\begin{array}{l}V_{p c} \\
(t)\end{array}$ & $\begin{array}{l}V_{\text {ri }} \\
(t)\end{array}$ & $\begin{array}{l}V_{c} \\
(t)\end{array}$ & $\begin{array}{l}V_{s} \\
(t)\end{array}$ & $\begin{array}{l}\mathbf{V}_{\mathbf{u}} \\
(\mathbf{t})\end{array}$ & $\begin{array}{l}V_{p p} \\
(t)\end{array}$ & $\begin{array}{c}\mathbf{V}_{\text {uef }} \\
(\mathbf{t})\end{array}$ \\
\hline TASCV3a0-R1c & 3.95 & 0.00 & 3.95 & 17.42 & 21.63 & 0.26 & 21.37 & 2.11 & 0.00 & 2.11 & 19.56 & 21.99 & 0.32 & 21.67 \\
\hline TASCV3a1-R1c & - & 4.12 & 2.45 & 17.71 & 20.40 & 0.25 & 20.16 & - & 4.94 & - & 18.69 & 18.07 & 0.37 & 17.71 \\
\hline TASCV3a2-R1c & - & 9.86 & 6.44 & 13.36 & 20.07 & 0.27 & 19.80 & - & 9.85 & 1.73 & 19.56 & 21.56 & 0.27 & 21.29 \\
\hline TASCV3a3-R1c & - & 9.60 & 2.67 & 19.08 & 21.95 & 0.19 & 21.75 & - & 13.13 & 8.74 & 13.19 & 22.23 & 0.30 & 21.93 \\
\hline TASCV3a4-R1c & - & 12.97 & 7.18 & 10.66 & $17.93 *$ & 0.09 & $17.84^{*}$ & - & 11.70 & 4.48 & 14.93 & $19.50 *$ & 0.09 & $19.41 *$ \\
\hline
\end{tabular}


También cabe señalar que, a momento positivo, el cortante máximo o último en trabes acarteladas se alcanza en un estado donde el concreto cercano al refuerzo longitudinal superior se encontraba prácticamente totalmente agrietado (por ejemplo, figura 11), por lo que la sección crítica se encuentra a flexiotensión y, por ello, no contribuye $V_{p c}$ (tabla 2). En cambio, para momento negativo, en general la fuerza cortante última en trabes acarteladas se presentó en un estado donde el concreto cercano al refuerzo longitudinal inferior se mantiene lo suficientemente saludable como para estar en un estado neto de flexocompresión (por ejemplo, figura 11). Sin embargo, del análisis de los valores obtenidos para $V_{p c}$ (tabla 1) se concluye que, en el instante de la carga última, la contribución del concreto se reduce a medida que aumenta el ángulo de acartelamiento $\alpha$, lo cual también se observa en la figura 10. Para ilustrar mejor lo discutido líneas arriba, en la figura 11 se muestran las ubicaciones del eje neutro al cortante $V_{u}$ para momentos positivo y negativo, para los puntos ubicados en negro y que corresponden a lecturas sanas de los strain gages ubicados en el refuerzo longitudinal superior e inferior. El eje neutro se calculó bajo la hipótesis de la sección plana a partir de las lecturas de los deformímetros ubicados en el acero longitudinal, como se ha indicado con anterioridad. En los croquis de la figura 11 se sombrea la zona de compresión y se indica con línea punteada en color rojo la ubicación teórica del eje neutro a flexión pura de la sección transversal, calculado conforme al bloque equivalente de esfuerzos del reglamento ACI 318. Se puede deducir de estas gráficas que, aún a la carga última, existe un acoplamiento importante a flexión y carga axial en las trabes acarteladas, particularmente cuando el refuerzo longitudinal inclinado trabaja a compresión (momento negativo), y que este acoplamiento varía con respecto al eje longitudinal, lo que coincide con lo reportado por Hou et al. (2015) en sus ensayes ante carga estática monótona creciente, al presentar perfiles de distribuciones de deformaciones unitarias en el concreto antes de la carga última a distintas profundidades (alturas) del peralte de la sección transversal, en distintas ubicaciones a lo largo de las cartelas, medidos estos perfiles con deformímetros para el concreto.

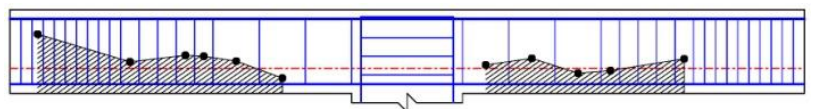

a) momento negativo, TASCV3 $\alpha 0-\mathrm{R} 1 \mathrm{c}$

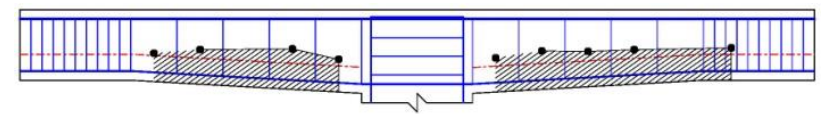

c) momento negativo, TASCV3 $\alpha 1-\mathrm{R} 1 \mathrm{c}$

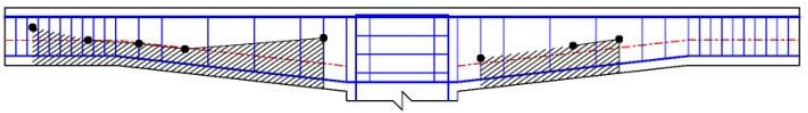

e) momento negativo, TASCV3 $\alpha 2-\mathrm{R} 1 \mathrm{c}$

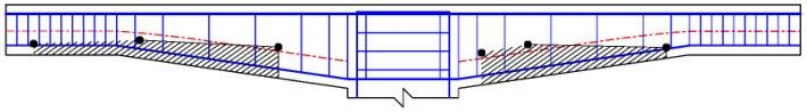

g) momento negativo, TASCV3a3-R1c

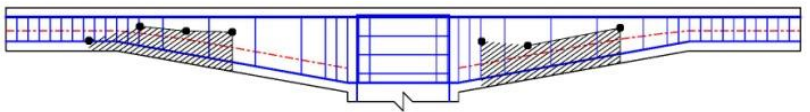

i) momento negativo, TASCV3 $\alpha 4-\mathrm{R} 1 \mathrm{c}$

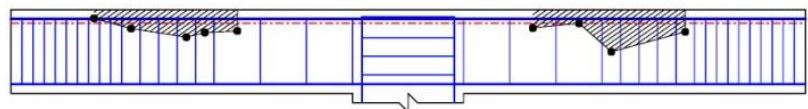

b) momento positivo, TASCV3 $\alpha 0-\mathrm{R} 1 \mathrm{c}$

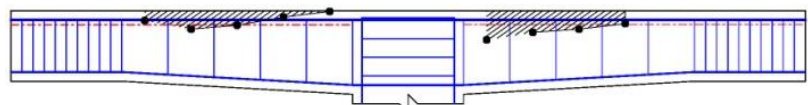

d) momento positivo, TASCV3 $\alpha 1-\mathrm{R} 1 \mathrm{c}$

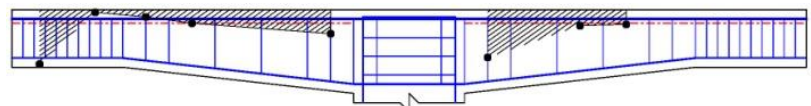

f) momento positivo, TASCV3 $\alpha 2-\mathrm{R} 1 \mathrm{c}$

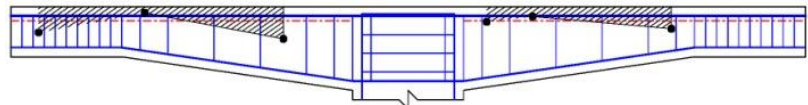

h) momento positivo, TASCV3 $\alpha 3-\mathrm{R} 1 \mathrm{c}$

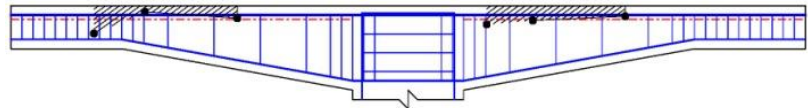

j) momento positivo, TASCV3 $\alpha 4-R 1 \mathrm{c}$

Figura 11. Ubicación del eje neutro experimental al cortante último $V_{u}$, espécimen en estudio 


\section{Mejoras de diseño con base experimental}

\section{Contribución del refuerzo inclinado}

Con la finalidad de mejorar las propuestas de ecuaciones de diseño, se estimaron los momentos flexionantes $\left(M_{\text {exp }}\right)$ efectivamente desarrollados a la carga máxima o última, $V_{u}$, en las secciones críticas por cortante (figuras $4 \mathrm{~b}$ a $8 \mathrm{~b}$ ) de las trabes acarteladas TASCV3 $\alpha \mathrm{i}-\mathrm{R} 1 \mathrm{c}$, que entre otros factores ya incluyen pérdidas por adherencia (figuras $4 \mathrm{a}$ a $8 \mathrm{a}$ ), y se normalizaron con respecto al momento flexionante nominal $\left(M_{n}\right)$ como si esa misma sección pudiera desarrollar una falla por flexión pura. Los cocientes $M_{\text {exp }} / M_{n}$ obtenidos se grafican contra $\tan \alpha$ y se muestran en la figura 12 , donde también se incluyen los obtenidos previamente (Tena-Colunga et al. 2008) para los experimentos de MacLeod y Houmsi (1994) para trabes acarteladas de concreto reforzado continuas sin refuerzo por cortante. En la figura 12 se tomó el ángulo $\alpha$ como positivo para trabes acarteladas que aumentan su peralte hacia el nudo. En la figura 12 también se identifica el letrero "Diseno" a la ecuación (ec. 4) propuesta para su diseño (Urbina 2013, Tena et al. 2014 y 2017), y se presentan la curva de regresión de los datos experimentales ahí indicados, más una propuesta mejorada de diseño, como se discute más adelante.

$$
M_{c r}=0.85 M_{n}(1+1.6 \tan \alpha) \leq M_{n}
$$

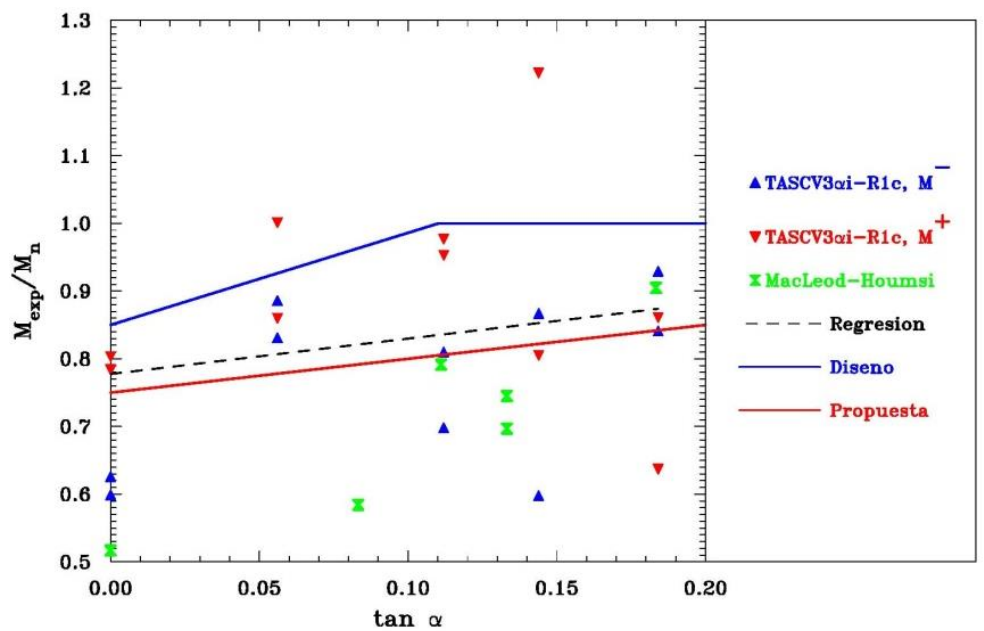

Figura 12. Contribución indirecta del refuerzo longitudinal inclinada a la resistencia a cortante de trabes acarteladas (ecuación de momento) en condiciones de continuidad

Se pueden hacer las siguientes observaciones de la figura 12: a) para la misma trabe acartelada, el cociente $M_{\text {exp }} / M_{n}$ es mayor para momento positivo que para momento negativo, dado que el refuerzo longitudinal inclinado desarrolló deformaciones unitarias proporcionalmente más altas, b) existen algunos cocientes $M_{\text {exp }} / M_{n}$ mayores a 1.0 para momento positivo, dado que el momento máximo se desarrolló en flexocompresión antes que se presentaran problemas por adherencia, c) como se esperaba (Tena et al. 2017), la ecuación propuesta para el diseño (ec. 4) sobrestimó a la mayoría de los momentos máximos desarrollados y, d) a pesar de la dispersión obtenida en los ensayes realizados, se puede obtener una regresión lineal útil para fines prácticos. Así, y basados en la regresión lineal de los datos experimentales ahí mostrados, se propone la ecuación 5 para estimar el momento flexionante de diseño en la sección crítica $\left(M_{c r}\right)$ para trabes acarteladas de concreto reforzado donde el diagrama de momento y el peralte de la cartela crecen en la misma dirección, y que se indica en la figura 11 como "propuesta". En la regresión estadística se obtuvo un coeficiente de variación de 0.184 para $M_{c r}$ asociado a la ecuación 5 . 


$$
M_{c r}=0.75 M_{n}(1+\tan \alpha) \leq M_{n}
$$

\section{Ángulo de inclinación de la grieta principal por cortante}

Se midieron los ángulos de inclinación de la grieta principal por cortante con respecto al plano horizontal $(\theta)$, tanto a momento negativo como positivo, como se ilustra en la figura 13. Posteriormente, se incluyeron en la base de datos experimentales confiables reportados en distintos estudios (MacLeod y Houmsi 1994, Archundia 2004 y 2013, Grande 2005 y 2009, Urbina 2013) y compilada por los autores, y se grafican contra el ángulo de acartelamiento $\alpha$, como se muestra en la figura 14 .

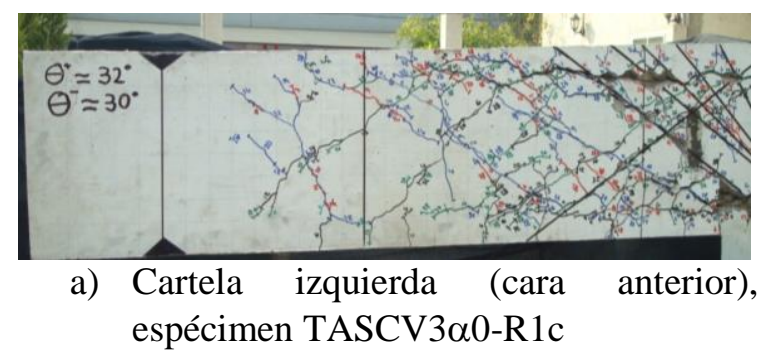

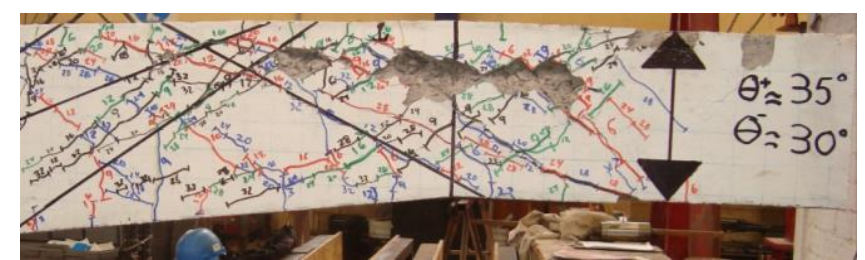

b) Cartela izquierda (cara posterior), espécimen TASCV $3 \alpha 3-R 1 c$

Figura 13.Grietas y ángulos principales por cortante medidos en las trabes en estudio

En la figura 14 se distinguen en color rojo los ángulos medidos en vigas simplemente apoyadas (y momento positivo) y en azul en vigas continuas (y momento negativo), con símbolos llenos se identifican los medidos en vigas con refuerzo transversal por cortante y con símbolos vacíos los medidos en trabes sin refuerzo por cortante. Se presenta también la regresión de los datos experimentales involucrados, y se identifica como " $45-\alpha$ " la ecuación previamente propuesta para estimar, de manera práctica, el ángulo de la grieta por cortante en función del ángulo de acartelamiento:

$$
\theta=45-\alpha
$$

que, por congruencia con el diseño de vigas de concreto reforzado por el método de las secciones, coincide con el ángulo propuesto en los reglamentos de concreto reforzado para trabes prismáticas $(\theta=$ $45^{\circ}$ ) cuando $\alpha=0$.

Como se aprecia de la figura 14, la ecuación previamente propuesta (ec. 6) es todavía válida, pero conservadora, dado que a medida que aumenta $\theta$, se subestima la contribución de los estribos en la resistencia a cortante. Una mejora basada en la nueva regresión estadística sería proponer la ecuación 7 también identificada en esa figura, la cual tiene un coeficiente de variación 0.191 para $\theta$ con base en los datos experimentales disponibles y graficados también en la figura 14:

$$
\theta=39-0.6 \alpha
$$

Sin embargo, emplear esta ecuación implicaría que en los reglamentos de concreto reforzado se reconozca finalmente que, estadísticamente, el ángulo de inclinación de la grieta por cortante en vigas prismáticas de concreto reforzado es distinto a $45^{\circ} \mathrm{y}$, generalmente, es menor a este valor para vigas con refuerzo a cortante. 


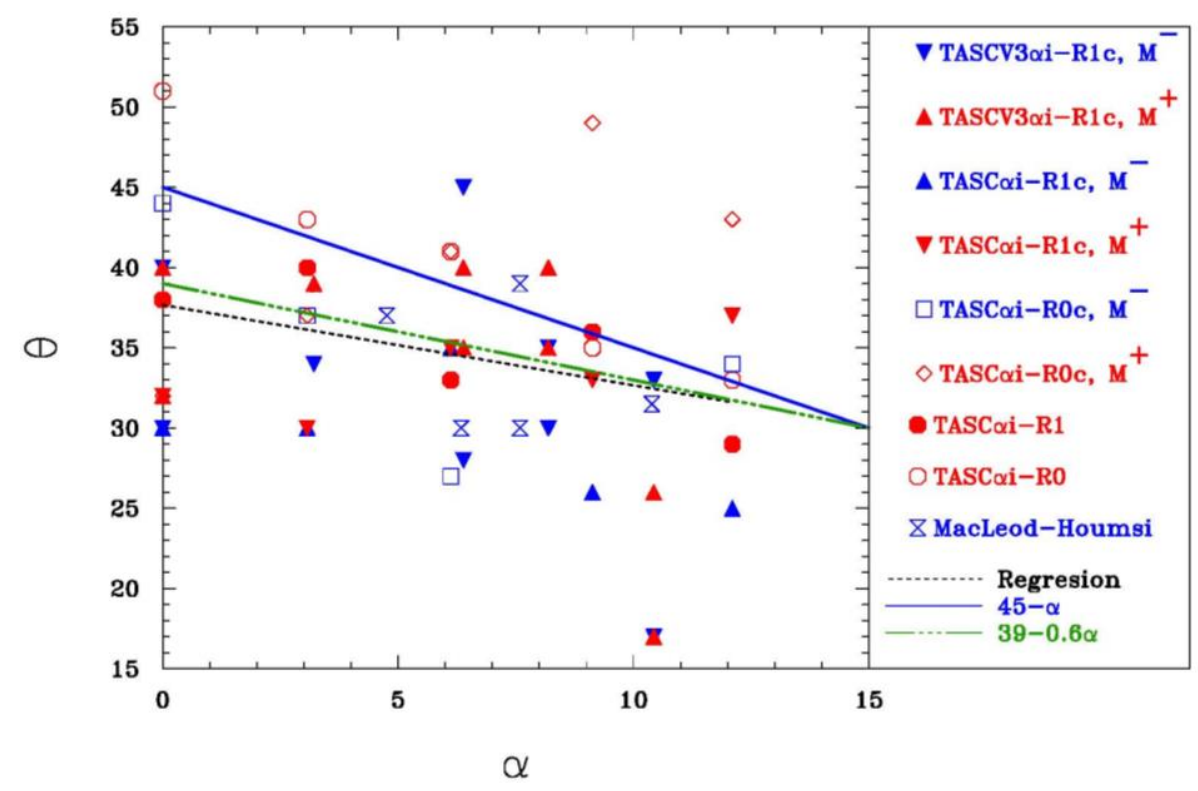

Figura 14. Ángulos medidos con respecto a un plano horizontal $(\theta)$ de las grietas principales por cortante en trabes acarteladas vs ángulo de acartelamiento $(\alpha)$

Con la finalidad de tener un mejor entendimiento de cómo influyen las distintas variables en estudio en el ángulo de inclinación de la grieta por cortante, se reagruparon los datos experimentales disponibles de la siguiente manera. Se dividieron: 1) por condiciones de apoyo en: simplemente apoyadas (figura 15a) o continuas (figura 15b), 2) por el tipo de refuerzo por cortante en: sin refuerzo por cortante (figura 15c) y con refuerzo por cortante (figura 15d), 3) por el tipo de momento flexionante en: momento positivo (figura 15e) y momento negativo (figura 15f) y, 4) por el tipo de carga en: estática monótona creciente (figura $15 \mathrm{~g}$ ) y cíclica (figura 15h). Para facilitar las interpretaciones, se respetaron los colores y símbolos usados en la figura 14, así como las ecuaciones propuestas, pero las curvas de regresión mostradas en la figura 15 corresponden exclusivamente a los datos experimentales ahí mostrados.

Se pueden hacer las siguientes observaciones de las gráficas mostradas en la figura 15:

1) En términos generales, los ángulos de inclinación de la grieta por cortante son mayores en condiciones de apoyo simple (figura 15a) que en condición de continuidad (figura 15b), lo que indica que, en general, la contribución de los estribos a la resistencia a cortante es más importante en una viga continua que en una viga simplemente apoyada. Esto parece cumplirse también para las vigas prismáticas, aunque en ese caso los datos que aumentan al ángulo $\theta$ son los de las vigas continuas sin refuerzo por cortante (figura 15a). Dado que las ecuaciones de diseño disponibles en la mayoría de los reglamentos de concreto reforzado se han basado en los resultados de ensayes de vigas prismáticas simplemente apoyadas, estos datos sugieren que, para fines prácticos, el diseño por cortante de vigas continuas pudiera ser conservador empleando esas ecuaciones.

2) Se comprueba que, generalmente, el ángulo de inclinación de la grieta principal a cortante es mayor para trabes sin refuerzo por cortante (figura 15c) que para trabes con refuerzo por cortante (figura 15d), algo que se ha observado ampliamente en vigas prismáticas. 


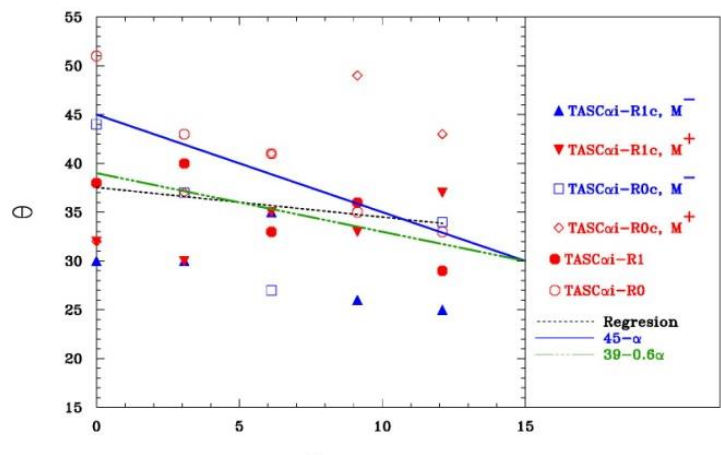

a) simplemente apoyadas

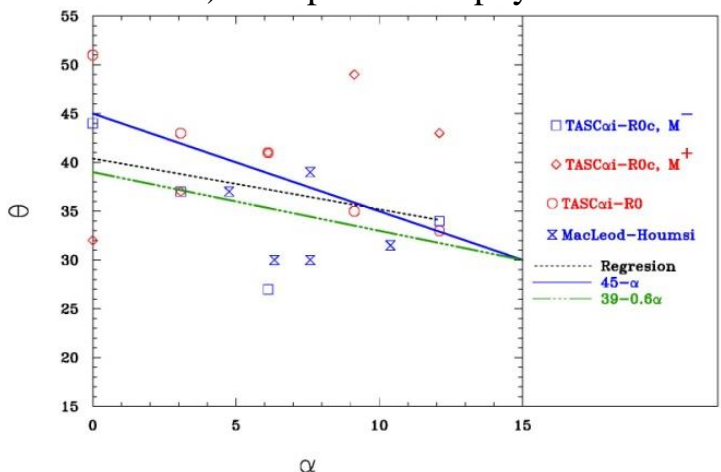

c) sin refuerzo por cortante

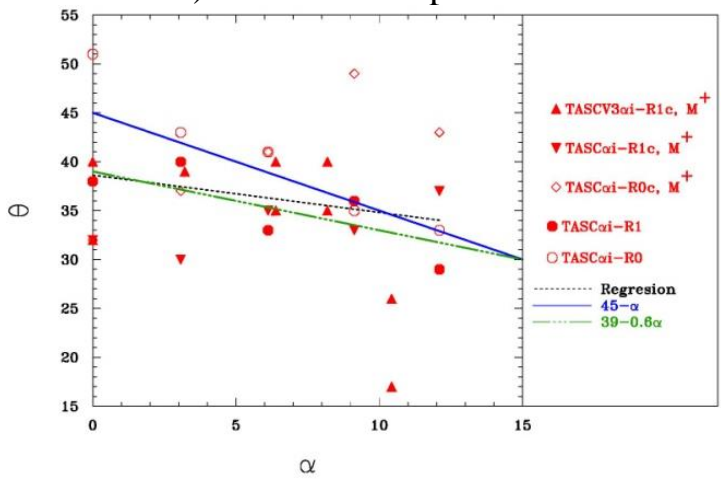

e) momento positivo

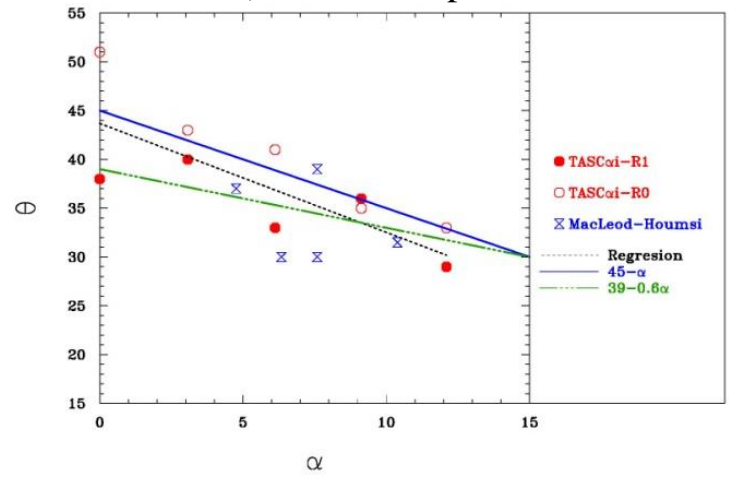

g) carga estática monótona creciente

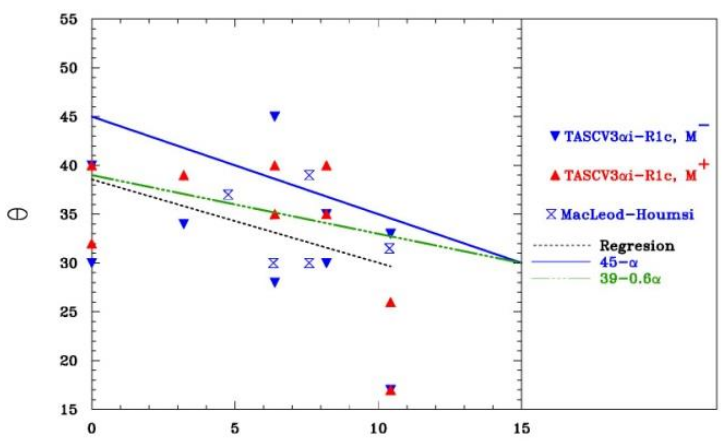

b) continuas (voladizos)

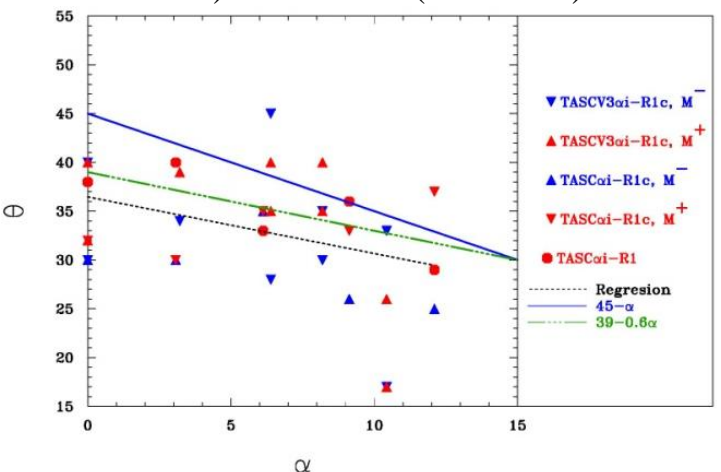

d) con refuerzo por cortante

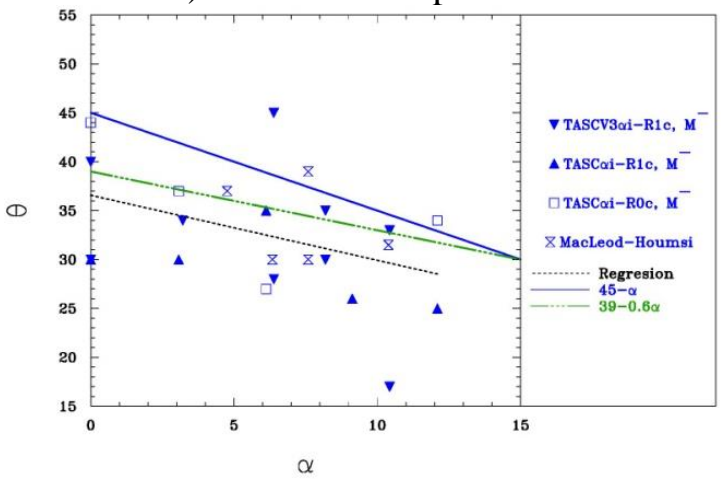

f) momento negativo

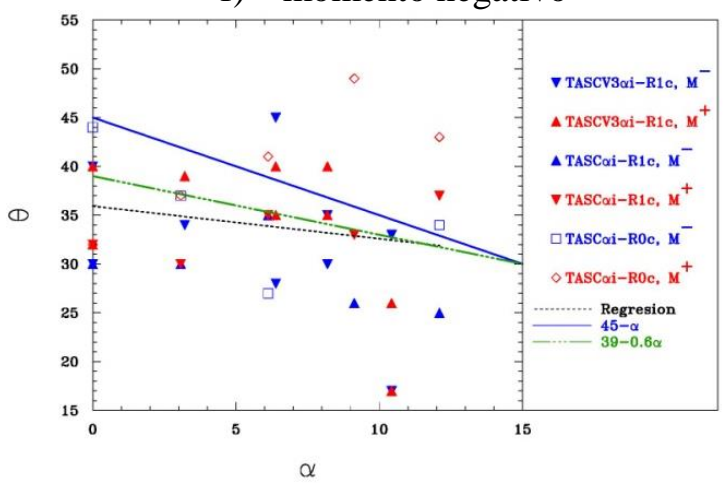

h) carga cíclica

Figura 15. Ángulos medidos con respecto a un plano horizontal $(\theta)$ de las grietas principales por cortante en trabes acarteladas vs ángulo de acartelamiento $(\alpha)$ 
3) Parece ser que los ángulos de inclinación de la grieta pudieran tener alguna dependencia del momento flexionante (figuras 15e y 15f); sin embargo, dado que la mayor parte de los datos obtenidos para momento positivo son en vigas simplemente apoyadas, las regresiones tienden a coincidir (figuras 15a y 15e). Lo mismo sucede para momento negativo, pues todos los datos experimentales provienen de una condición de continuidad (figuras 15b y 15f). Sin embargo, si uno se concentra en apoyarse con las gráficas que discriminan la presencia o no de refuerzo por cortante (figuras $15 \mathrm{c}$ y $15 \mathrm{~d}$ ), y ubica que en azul están graficados los resultados asociados a momentos negativos y en rojo los asociados a momentos positivos, sí se aprecia que, en general, los ángulos de inclinación de las grietas son menores para momento negativo que para momento positivo.

4) Parece ser que el ángulo de la inclinación de las grieta principal depende del tipo de carga aplicada, resultando en general mayor para cargas monótonas (figura $15 \mathrm{~g}$ ) que en cargas cíclicas (figura 15h), donde además se observa una mayor dispersión de los datos experimentales.

5) Se aprecia que la ecuación propuesta anteriormente (ec. 6) es muy razonable para los siguientes casos: vigas simplemente apoyadas (figura 15a), vigas sin refuerzo por cortante (figura 15c), vigas a momento positivo (figura 15e) y cargas monótonas crecientes (figura $15 \mathrm{~g}$ ). Sin embargo, es conservadora para los siguientes escenarios: vigas continuas (figura 15b), vigas con refuerzo por cortante (figura 15d), vigas a momento negativo (figura 15f) y cargas cíclicas (figura 15h).

\section{ESTIMACIÓN DE LA RESISTENCIA AL CORTE CON FINES DE DISEÑO}

En el ejercicio profesional se requieren de métodos simples para estimar razonablemente la resistencia a cortante de trabes acarteladas, para poder diseñarlas adecuadamente evitando esta falla no deseada y poder asegurar que trabajen eficientemente a flexión. A continuación se presentarán dos propuestas de diseño, que están en congruencia con los métodos comúnmente utilizados en la práctica de diseño y en los reglamentos de diseño de elementos de concreto reforzado: el método de la sección crítica y el método de puntales y tensores. Las ecuaciones desarrolladas y propuestas son confrontadas con los resultados experimentales, para dar un marco de referencia.

\section{Método de la sección crítica}

El método de la sección crítica parte de plantear las ecuaciones de equilibrio en la sección crítica por cortante y, de hecho, fue con el que se estimaron las resistencias experimentales en secciones anteriores. Los autores han propuesto con anterioridad, basándose en los planteamientos pioneros de Mörsch (1952), Debaiky y El-Niema (1982) y MacLeod y Houmsi (1994), una ecuación muy robusta, con base experimental, para estimar la resistencia a cortante de trabes acarteladas simplemente apoyadas que fallan a cortante (Tena-Colunga et al. 2008) y, como se demostró en ese artículo, con ella se obtienen las mejores correlaciones con la base de datos experimentales confiables de los experimentos propios y reportados por otros, comparando con otras propuestas previas, como se reporta con detalle en TenaColunga et al. (2008). También resultó ser confiable para predecir la resistencia última de este tipo de trabes ante cargas cíclicas (Archundia-Aranda et al. 2013). Además, las ecuaciones propuestas tienen la virtud que coinciden con las ecuaciones de diseño del ACI-318 para vigas prismáticas cuando $\alpha=0$, facilitando su probable inserción en un reglamento de diseño de concreto reforzado, a juicio de los profesionales encargados en hacer estas evaluaciones. 
Aunque el planteamiento general se presenta en el artículo compañero (Tena et al. 2017), pues con base en una adaptación de este procedimiento se realizó el prediseño de los especímenes de prueba aquí reportados, con fines exclusivamente de transparencia y para que todos los lectores interesados tengan certidumbre total de las ecuaciones empleadas, y no sólo los más intuitivos, se repetirán algunas ecuaciones y notaciones a continuación.

Por lo tanto, la estimación del cortante último de diseño de trabes acarteladas en condiciones de continuidad se hace de la siguiente manera. La resistencia nominal a cortante de las trabes, $V_{n}$, se determina conforme a la ecuación 1 y la contribución total del concreto, $V_{c}$, conforme a la ecuación 2 . La contribución nominal del concreto $V_{p c}$, se hace conforme lo propone el reglamento ACI-318, dada por la ecuación 8:

$$
V_{p c}=\left(0.5 \sqrt{f_{c}^{\prime}}+176 \rho_{w} \frac{V_{n} d_{c r}}{M_{n}}\right) b d_{c r}
$$

donde $b$ es el ancho de la sección transversal, $f_{c}^{\prime}$ es la resistencia nominal a compresión del concreto, $\rho_{w}$ es la cuantía de refuerzo longitudinal a flexión, $V_{n}$ y $M_{n}$ son, respectivamente, el cortante nominal y momento nominal aplicados a la sección crítica, y $d_{c r}$ es el peralte crítico por cortante en las cartelas y se calcula de la siguiente forma (Archundia 2004, Tena-Colunga et al. 2008):

$$
d_{c r}=d_{\text {min }}[1+1.35 \tan \alpha] \leq\left[\left(\frac{h_{\max } h_{\min }-h_{\max }^{2}}{2 l_{h}}+h_{\max }\right)-r\right]
$$

donde $\alpha$ es el ángulo de acartelamiento, $d_{\min }$ es el peralte efectivo menor en las cartelas, $h_{\max } \mathrm{y} h_{\min }$ es el peralte total máximo y mínimo en las cartelas, $l_{h}$ es la longitud de acartelamiento y $r$ es el recubrimiento del concreto para el refuerzo longitudinal.

Para calcular la contribución del acero de refuerzo transversal en la resistencia a la fuerza cortante, $V_{s}$, se utilizó la ecuación propuesta en Tena-Colunga et al. (2008):

$$
V_{s}=\frac{A_{v} f_{y v} d_{c r}}{s \tan \theta}
$$

donde $\theta$ es el ángulo de inclinación de la grieta principal por cortante (por ejemplo, ecuaciones 6 o 7) y $A_{v}$ es la suma de las áreas de las ramas de los estribos separados una distancia, $s$. El peralte, $d_{c r}$, se calcula conforme a la ecuación (9), y $f_{y v}$ es el esfuerzo de fluencia del acero del refuerzo transversal.

La contribución del acero de refuerzo longitudinal inclinado, $V_{r i}$, se calcula conforme a la ecuación 3. Como se ha mencionado en el artículo compañero (Tena et al. 2017), el diseño se hizo estimando $M_{c r}$ de manera no conservadora conforme a la ecuación 4, pero con los datos experimentales disponibles ya se puede hacer una propuesta razonable empleando la ecuación 5.

Para la estimación de las resistencias, se empleó la información experimental, como las propiedades del concreto determinadas del ensaye de cilindros a la edad del ensaye del espécimen (lado izquierdo y derecho), que se reportan en la tabla 3 . Se consideró también el esfuerzo de fluencia $f_{y}=4,348 \mathrm{~kg} / \mathrm{cm}^{2}$ para el acero de refuerzo longitudinal y $f_{y v}=4,592 \mathrm{~kg} / \mathrm{cm}^{2}$ para el acero de refuerzo transversal. 
Arturo Tena Colunga, Luis A. Urbina Californias y Hans I. Archundia Aranda

Tabla 3. Propiedades del concreto medidas a la edad del ensaye experimental

\begin{tabular}{|c|c|c|c|c|c|c|}
\hline \multirow{2}{*}{ Espécimen } & \multicolumn{3}{|c|}{ Cartela (viga) izquierda } & \multicolumn{3}{|c|}{ Cartela (viga) derecha } \\
\hline & $f_{c}^{\prime}\left(\mathrm{kg} / \mathrm{cm}^{2}\right)$ & $E_{c}\left(\mathrm{~kg} / \mathrm{cm}^{2}\right)$ & $f_{r}\left(\mathrm{~kg} / \mathrm{cm}^{2}\right)$ & $f_{c}^{\prime}\left(\mathrm{kg} / \mathrm{cm}^{2}\right)$ & $E_{c}\left(\mathrm{~kg} / \mathrm{cm}^{2}\right)$ & $f_{r}\left(\mathrm{~kg} / \mathrm{cm}^{2}\right)$ \\
\hline TASCV3 $\alpha 0-R 1 c$ & 292.25 & 125,685 & 32.51 & 310.38 & 128,891 & 29.80 \\
\hline TASCV3 $\alpha 1-R 1 c$ & 269.08 & 130,628 & 28.03 & 272.01 & 123,893 & 28.86 \\
\hline TASCV3 $\alpha 2-R 1 c$ & 286.48 & 119,648 & 21.09 & 283.92 & 117,833 & 22.30 \\
\hline TASCV3 $\alpha 3-R 1 c$ & 255.47 & 124,279 & 24.65 & 263.63 & 120,967 & 22.79 \\
\hline TASCV3 $\alpha 4-R 1 c$ & 326.23 & 122,525 & 20.82 & 321.93 & 124,604 & 19.32 \\
\hline
\end{tabular}

Se hicieron dos ejercicios de comparación de las ecuaciones con el método de las secciones, y que involucran básicamente tanto los resultados experimentales, como las mejoras con base experimental. Así, para determinar $V_{r i}$, se utilizó siempre la propuesta que ya incorpora la información experimental para estimar $M_{c r}$, y que es la ecuación 5. La variable que se evalúa es el ángulo de inclinación de la grieta principal por cortante, $\theta$. Para ello, se consideraron dos escenarios: 1) utilizando la ecuación 6 propuesta en trabajos anteriores, que hace $100 \%$ compatible a la propuesta con el método de las secciones de los reglamentos internacionales de concreto para vigas prismáticas y, 2) utilizando la ecuación 7 , que es menos conservadora, pero más apegada a la base experimental disponible.

Los resultados así obtenidos se reportan en las tablas 4 a 7, donde se comparan con los cortantes últimos efectivos $V_{\text {uef }}$ determinados experimentalmente y mostrados en las tablas 1 y 2 . Cabe señalar que en las tablas 4 a 7 se indican con un asterisco (*) los cocientes $V_{n} / V_{\text {uef }}$ calculados para el espécimen TASCV $3 \alpha 4-R 1 c$, ya que $V_{u e f}$ no corresponde al cortante último, por la falla prematura del vértice derecho de la cartela, como ya se ha explicado.

De la información reportada en las tablas 4 y 5 , se pueden hacer las siguientes observaciones, en términos generales, cuando se considera $\theta=45-\alpha$ : a) la estimación del cortante nominal de las trabes acarteladas $V_{n}$ es razonablemente buena y conservadora para momento negativo (tabla 4). Las peores estimaciones se obtuvieron para las trabes prismáticas, donde la ecuación coincide con la propuesta por el reglamento ACI-318. De hecho, si se toma el único valor de la trabe TASCV3 $\alpha 4-\mathrm{R} 1 \mathrm{c}$ que corresponde a la falla (cartela derecha), la estimación es buena, aunque no conservadora, b) la estimación del cortante nominal de las trabes acarteladas $V_{n}$ es razonablemente buena, pero algo no conservadora para momento positivo (tabla 5), c) la estimación del cortante aportado por el acero longitudinal inclinado, $V_{r i}$, es razonable para momento negativo si se compara con la determinada experimentalmente (tablas 1 y 4 ). La estimación de $V_{r i}$ es razonable pero conservadora para momento positivo, dado que se obtuvieron resistencias más grandes de los datos experimentales (tablas 2 y 5) y, d) como se esperaba, la contribución del acero de refuerzo transversal es conservadora y se subestima notablemente si se compara con respecto a los valores determinados experimentalmente, sobre todo a momento negativo, particularmente para ángulos de acartelamiento de seis grados o menores (tablas 1 y 4), como consecuencia de proponer $\theta=45$ $\alpha$ (figuras 14 y 15f) para mantener a las ecuaciones de diseño compatibles al $100 \%$ con las propuestas por el ACI-318 para el diseño de vigas prismáticas.

Es por ello que se decidió que sería un ejercicio académico interesante evaluar cuánto mejoran a momento negativo y empeoran a momento positivo las predicciones si se emplea un ángulo de la grieta principal por cortante $\theta=39-0.6 \alpha$, más cercano a la regresión de todos los datos experimentales confiables disponibles (figura 14). Estos resultados se reportan en las tablas 6 y 7, donde se confirma que utilizando esta propuesta para $\theta$ se mejoran las aproximaciones para momento negativo (tablas 1 y 6 ), sobre todo donde más se requieren y que son los ángulos inferiores a seis grados, donde las aproximaciones mejoran de un $12.9 \%$ máximo para las trabes prismáticas a un $5.2 \%$ mínimo para las 
trabes de seis grados (tablas 4 y 6). En contraste y como se esperaba, para momento positivo la aproximación disminuye, pues aumentan las sobrestimaciones (tablas 5 y 7 ).

Tabla 4. Cortantes nominales estimados con las ecuaciones de diseño cuando $\theta=45-\alpha$, momento negativo

\begin{tabular}{|c|c|c|c|c|c|c|c|c|c|c|c|c|}
\hline \multirow[t]{2}{*}{ Espécimen } & \multicolumn{6}{|c|}{ Cartela (viga) izquierda } & \multicolumn{6}{|c|}{ Cartela (viga) derecha } \\
\hline & $\begin{array}{l}V_{p c} \\
(t)\end{array}$ & $\begin{array}{l}V_{r i} \\
(t)\end{array}$ & $\begin{array}{l}\mathbf{V}_{\mathrm{c}} \\
(\mathbf{t})\end{array}$ & $\begin{array}{l}V_{s} \\
(t)\end{array}$ & $\begin{array}{l}V_{n} \\
(t)\end{array}$ & $\mathbf{V}_{\mathbf{n}} / \mathbf{V}_{\text {uef }}$ & $\begin{array}{l}V_{p c} \\
\text { (t) }\end{array}$ & $\begin{array}{l}V_{r i} \\
(t)\end{array}$ & $\begin{array}{l}\mathbf{V}_{\mathrm{c}} \\
(\mathbf{t})\end{array}$ & $\begin{array}{l}\mathbf{V}_{\mathrm{s}} \\
(\mathrm{t})\end{array}$ & $\begin{array}{l}V_{n} \\
(t)\end{array}$ & $\overline{V_{n} / V_{\text {uef }}}$ \\
\hline TASCV3 $\alpha 0-R 1 c$ & 9.47 & 0.00 & 9.47 & 10.43 & 19.90 & 0.669 & 9.74 & 0.00 & 9.74 & 10.43 & 20.18 & 0.651 \\
\hline TASCV3 $\alpha 1-R 1 c$ & 8.41 & 5.04 & 13.44 & 10.36 & 23.80 & 0.816 & 8.45 & 5.04 & 13.49 & 10.36 & 23.85 & 0.859 \\
\hline TASCV3 $\alpha 2-R 1 c$ & 7.63 & 9.19 & 16.81 & 9.78 & 26.59 & 0.754 & 7.60 & 9.18 & 16.78 & 9.78 & 26.56 & 0.846 \\
\hline TASCV3 $\alpha 3-R 1 c$ & 6.60 & 10.98 & 17.59 & 9.16 & 26.75 & 0.870 & 6.69 & 11.00 & 17.69 & 9.16 & 26.85 & 0.881 \\
\hline TASCV3a4-R1c & 6.53 & 13.06 & 19.59 & 8.51 & 28.10 & $1.315^{*}$ & 6.50 & 13.05 & 19.55 & 8.51 & 28.05 & 1.085 \\
\hline
\end{tabular}

Tabla 5. Cortantes nominales estimados con las ecuaciones de diseño cuando $\theta=45-\alpha$, momento positivo

\begin{tabular}{|c|c|c|c|c|c|c|c|c|c|c|c|c|}
\hline \multirow[t]{2}{*}{ Espécimen } & \multicolumn{6}{|c|}{ Cartela (viga) izquierda } & \multicolumn{6}{|c|}{ Cartela (viga) derecha } \\
\hline & $\begin{array}{l}V_{p c} \\
(t)\end{array}$ & $\begin{array}{l}V_{\text {ri }} \\
(t)\end{array}$ & $\begin{array}{l}V_{c} \\
(t)\end{array}$ & $\begin{array}{l}V_{s} \\
(t)\end{array}$ & $\begin{array}{l}V_{n} \\
(t)\end{array}$ & $\mathbf{V}_{\mathbf{n}} / \mathbf{V}_{\text {uef }}$ & $\begin{array}{l}\mathbf{V}_{\mathbf{p c}} \\
(\mathbf{t})\end{array}$ & $\begin{array}{l}V_{\text {ri }} \\
(t)\end{array}$ & $\begin{array}{l}V_{c} \\
(t)\end{array}$ & $\begin{array}{l}\mathbf{V}_{\mathrm{s}} \\
(\mathrm{t})\end{array}$ & $\begin{array}{l}V_{n} \\
(t)\end{array}$ & $\mathbf{V}_{\mathbf{n}} / \mathbf{V}_{\text {uef }}$ \\
\hline TASCV3a0-R1c & 9.45 & 0.00 & 9.45 & 10.43 & 19.88 & 0.930 & 9.72 & 0.00 & 9.72 & 10.43 & 20.15 & 0.930 \\
\hline TASCV3a1-R1c & 8.30 & 3.46 & 11.75 & 10.36 & 22.11 & 1.097 & 8.34 & 3.46 & 11.80 & 10.36 & 22.16 & 1.251 \\
\hline TASCV3 $\alpha 2-R 1 c$ & 7.44 & 6.34 & 13.78 & 9.78 & 23.56 & 1.190 & 7.42 & 6.34 & 13.75 & 9.78 & 23.53 & 1.104 \\
\hline TASCV3 $\alpha 3-R 1 c$ & 6.37 & 7.65 & 14.02 & 9.16 & 23.18 & 1.066 & 6.46 & 7.65 & 14.11 & 9.16 & 23.27 & 1.061 \\
\hline TASCV3a4-R1c & 6.26 & 9.11 & 15.37 & 8.51 & 23.87 & $1.338 *$ & 6.22 & 9.10 & 15.32 & 8.51 & 23.83 & $1.228 *$ \\
\hline
\end{tabular}

Tabla 6. Cortantes nominales estimados con las ecuaciones de diseño cuando $\theta=39-0.6 \alpha$, momento negativo

\begin{tabular}{|c|c|c|c|c|c|c|c|c|c|c|c|c|}
\hline \multirow[t]{2}{*}{ Espécimen } & \multicolumn{6}{|c|}{ Cartela (viga) izquierda } & \multicolumn{6}{|c|}{ Cartela (viga) derecha } \\
\hline & $\begin{array}{l}V_{p c} \\
(t)\end{array}$ & $\begin{array}{l}V_{r i} \\
(t)\end{array}$ & $\begin{array}{l}V_{c} \\
(t)\end{array}$ & $\begin{array}{l}V_{s} \\
(t)\end{array}$ & $\begin{array}{l}\mathbf{V}_{\mathbf{n}} \\
(\mathbf{t})\end{array}$ & $\mathbf{V}_{\mathbf{n}} / \mathbf{V}_{\text {uef }}$ & $\begin{array}{l}\mathbf{V}_{\mathrm{pc}} \\
(\mathrm{t})\end{array}$ & $\begin{array}{l}V_{\text {ri }} \\
(t)\end{array}$ & $\begin{array}{l}V_{c} \\
(t)\end{array}$ & $\begin{array}{l}V_{s} \\
(t)\end{array}$ & $\begin{array}{l}V_{n} \\
(t)\end{array}$ & $\mathbf{V}_{\mathbf{n}} / \mathbf{V}_{\text {uef }}$ \\
\hline TASCV3a0-R1c & 9.59 & 0.00 & 9.59 & 12.88 & 22.47 & 0.755 & 9.86 & 0.00 & 9.86 & 12.88 & 22.75 & 0.734 \\
\hline TASCV3a1-R1c & 8.50 & 5.04 & 13.54 & 12.25 & 25.79 & 0.885 & 8.54 & 5.04 & 13.58 & 12.25 & 25.84 & 0.930 \\
\hline TASCV3 $\alpha 2-R 1 c$ & 7.70 & 9.19 & 16.89 & 11.08 & 27.97 & 0.794 & 7.67 & 9.18 & 16.85 & 11.08 & 27.93 & 0.890 \\
\hline TASCV3 3a3-R1c & 6.66 & 10.98 & 17.64 & 10.13 & 27.77 & 0.903 & 6.74 & 11.00 & 17.74 & 10.13 & 27.87 & 0.914 \\
\hline TASCV3a4-R1c & 6.57 & 13.06 & 19.63 & 9.12 & 28.74 & $1.345^{*}$ & 6.53 & 13.05 & 19.58 & 9.12 & 28.69 & 1.110 \\
\hline
\end{tabular}

Tabla 7. Cortantes nominales estimados con las ecuaciones de diseño cuando $\theta=39-0.6 \alpha$, momento positivo

\begin{tabular}{|c|c|c|c|c|c|c|c|c|c|c|c|c|}
\hline \multirow[t]{2}{*}{ Espécimen } & \multicolumn{6}{|c|}{ Cartela (viga) izquierda } & \multicolumn{6}{|c|}{ Cartela (viga) derecha } \\
\hline & $\begin{array}{l}\mathbf{V}_{\mathbf{p c}} \\
(\mathbf{t})\end{array}$ & $\begin{array}{l}V_{\mathbf{r i}} \\
(\mathbf{t})\end{array}$ & $\begin{array}{l}\mathbf{V}_{\mathbf{c}} \\
(\mathrm{t})\end{array}$ & $\begin{array}{l}V_{s} \\
(t)\end{array}$ & $\begin{array}{l}\mathbf{V}_{\mathbf{n}} \\
(\mathbf{t})\end{array}$ & $\mathbf{V}_{n} / \mathbf{V}_{\text {uef }}$ & $\begin{array}{l}V_{p c} \\
(t)\end{array}$ & $\begin{array}{l}V_{\text {ri }} \\
(t)\end{array}$ & $\begin{array}{l}\mathbf{V}_{\mathrm{c}} \\
(\mathrm{t})\end{array}$ & $\begin{array}{l}V_{s} \\
(t)\end{array}$ & $\begin{array}{l}V_{\mathbf{n}} \\
(\mathbf{t})\end{array}$ & $V_{n} / V_{\text {uef }}$ \\
\hline TASCV3a0-R1c & 9.56 & 0.00 & 9.56 & 12.88 & 22.45 & 1.051 & 9.84 & 0.00 & 9.84 & 12.88 & 22.72 & 1.048 \\
\hline TASCV3a2-R1c & 7.51 & 6.34 & 13.85 & 11.08 & 24.93 & 1.259 & 7.48 & 6.34 & 13.82 & 11.08 & 24.90 & 1.170 \\
\hline TASCV3a3-R1c & 6.42 & 7.65 & 14.07 & 10.13 & 24.20 & 1.113 & 6.51 & 7.65 & 14.16 & 10.13 & 24.29 & 1.108 \\
\hline TASCV3a4-R1c & 6.29 & 9.11 & 15.40 & 9.12 & 24.51 & $1.374 *$ & 6.26 & 9.10 & 15.36 & 9.12 & 24.47 & $1.261^{*}$ \\
\hline
\end{tabular}

Finalmente, dado que en el diseño por sismo más carga vertical de una viga continua va a dominar la condición del momento negativo sobre positivo para definir el refuerzo por cortante de la viga en sus extremos, parece recomendable diseñar trabes acarteladas bajo esta condición estimando al ángulo de la grieta principal por cortante $\theta$ conforme a la ecuación 6 , aunque los autores no estarían en desacuerdo en que se empleé la ecuación 5 para mantener congruencia con la filosofía de los reglamentos vigentes, hasta que se compile una base de datos experimentales más grande y extensa que permita definir una ecuación del tipo $\theta=\theta_{0}-a_{1} \alpha$, donde $\theta_{0}$ es el ángulo medio estadístico de la grieta principal por cortante para vigas prismáticas y $a_{l}$ es la pendiente media estadística de los datos experimentales de esa grieta obtenidos en trabes acarteladas. 


\section{Método de puntales y tensores}

Archundia (2013) desarrolló un método de diseño a cortante para trabes acarteladas de concreto reforzado basado en campos de esfuerzo y modelos de armadura, también conocido como modelos de puntales y tensores, que incorpora definiciones racionales para la sección crítica por cortante, la esbeltez y las regiones B-D (Bernoulli-Discontinuidad), cuyo aspectos más relevantes se presentan también en Archundia y Tena (2015a). A continuación se presentan sintéticamente las observaciones más importantes que definen las ecuaciones propuestas para diseño con base en el método de puntales y tensores.

Con base en los campos de esfuerzo que se desarrollan en la región B (de Bernoulli), el agrietamiento diagonal de una trabe acartelada tiene la proyección horizontal mostrada en la figura 16. Dentro de esta longitud se deben distribuir uniformemente los estribos que resisten la demanda de cortante. Estos campos de esfuerzo explican el por qué el agrietamiento diagonal de las TACR es más extenso que el de las prismáticas.

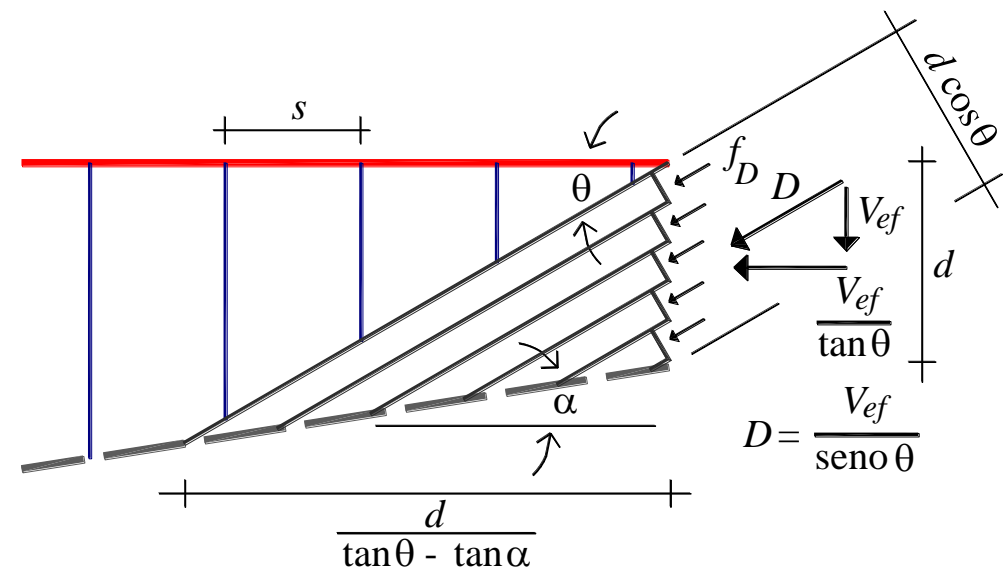

Figura 16. Campo de compresión diagonal en la región B de una trabe acartelada

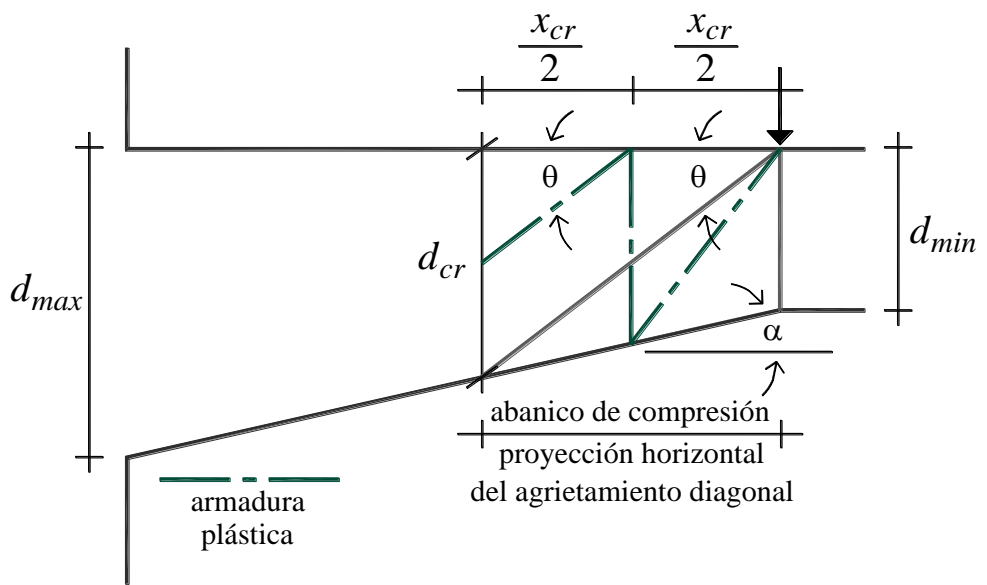

Figura 17. Ubicación de la sección crítica en una trabe acartelada con refuerzo transversal

Con base en ello, se definió la ubicación de la sección crítica de una trabe acartelada con refuerzo transversal (figura 17), y a partir de ahí, se demuestra que el diseño del acero de refuerzo transversal de la región B de las trabes acarteladas debe realizarse conforme con las ecuaciones 11 y 12 : 


$$
\begin{aligned}
V_{s} & =\frac{A_{v} f_{y v} d_{c r}}{s(\tan \theta-\tan \alpha)}\left(1-\frac{\tan \alpha}{2 \tan \theta}\right) \\
d_{c r} & =\frac{d_{\text {min }}}{1-\frac{\tan \alpha}{\tan \theta}} \leq d_{\max }\left(1-\frac{\tan \alpha}{\tan \theta}\right)
\end{aligned}
$$

donde la mayoría de los términos se ilustran en las figuras 16 y 17, o ya han sido descritos en secciones anteriores.

En la figura 18 se muestra la armadura elástica propuesta por Archundia (2013) para la región B de una trabe acartelada. Las demandas en la sección de análisis son el momento $\left(M_{a g r}\right)$ y el cortante $\left(V_{a g r}\right)$ justo antes del agrietamiento por tensión diagonal, y se descomponen en las fuerzas mostradas en el lado opuesto del corte $\left(T_{a g r}, C_{a g r}\right.$ y $\left.V_{c e f}\right)$. Al cortante efectivo $\left(V_{c e f}\right)$ se le agregó el subíndice "c" para relacionarlo con la resistencia del concreto. El ángulo de inclinación de los elementos que representan los campos de esfuerzo elástico es $\theta_{E}$, y no necesariamente tiene el valor de $\theta$. La sección de análisis corresponde a la ruptura completa del tensor de concreto debido al agrietamiento por tensión diagonal. Este agrietamiento inicia donde el tensor divide en dos partes iguales el peralte efectivo de la trabe, que corresponde al primer agrietamiento diagonal. Por lo tanto, el cortante del concreto conforme al modelo de la armadura elástica es el indicado en la ecuación 13:

$$
V c_{e f}=\frac{V_{a g r}-\frac{M_{a g r}}{d} \tan \alpha}{1+\frac{\tan \alpha}{2 \tan \theta_{E}}}
$$
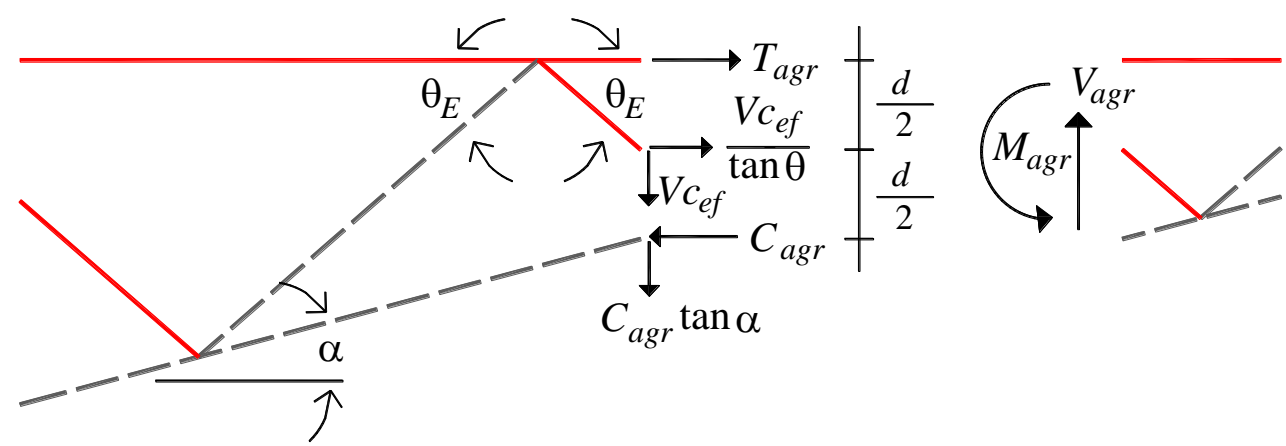

Figura 18. Modelo de armadura elástica para la región B de una trabe acartelada

La contribución del concreto $\left(V_{c}\right)$ propuesta por Archundia (2013) para trabes acarteladas de concreto reforzado tiene su origen tanto en la ecuación 13, como en una de las fórmulas semi-empíricas del ACI-318 para estimar la contribución del concreto de trabes prismáticas, y en cuyo desarrollo se tomó en cuenta la importancia que tiene el bloque de compresión no agrietado. Al combinarlas racionalmente se obtiene la ecuación 14, donde el signo positivo del cociente $\left|M_{a g r}\right| / d$ aplica cuando el peralte y el momento flexionante crecen en la misma dirección. Para usar la ecuación 14, se debe conocer el valor del 
momento asociado al agrietamiento diagonal $\left(M_{a g r}\right)$, así como el ángulo de inclinación $\left(\theta_{E}\right)$ del campo de esfuerzos elásticos.

$$
V_{c}=0.53 \sqrt{f_{c}^{\prime}} b d\left[1+\frac{\tan \alpha}{2 \tan \theta_{E}}\right] \pm \frac{M_{a g r} \mid}{d} \tan \alpha
$$

Por lo tanto, se aprecia a partir de comparar la ecuación 2 con la ecuación 14, uniformizando notación con el método de las secciones, observando de las figuras 16 a 18 que $d=d_{\min }$ y proponiendo que $\theta_{E} \approx \theta$, que igualando las ecuaciones 2 y 14 , entonces se tendría que:

$$
\begin{aligned}
& V_{p c}=0.53 \sqrt{f^{\prime}} b d_{\text {min }}\left[1+\frac{\tan \alpha}{2 \tan \theta}\right] \\
& V_{a g r}= \pm \frac{M_{a g r} \mid}{d_{\text {min }}} \tan \alpha
\end{aligned}
$$

Una de las "ventajas" (simplificaciones) de emplear la ecuación 15 para estimar $V_{p c}$ es que es independiente de la cuantía de refuerzo longitudinal principal a flexión, por lo que la estimación es igual a momento positivo que negativo (en contraste con la ecuación 8). De acuerdo con Nielsen (1999), el momento asociado al agrietamiento diagonal $\left(M_{a g r}\right)$ se puede calcular de manera sencilla, con un bloque recto alineado con la línea que une los extremos de la grieta teórica. Si en lugar del peralte total $(h)$, los cálculos se hacen de forma más correcta con el peralte crítico efectivo $\left(d_{c r}\right)$ de la trabe acartelada, a partir de la figura 17 se puede demostrar que el momento de agrietamiento diagonal se calcula con la ecuación 17, donde $f_{\text {tef }}$ es la resistencia efectiva a tensión del concreto y $b$ el ancho de la sección transversal:

$$
M_{\text {agr }}=0.5 f_{\text {tef }} b\left(\frac{d_{c r}}{\operatorname{sen} \theta}\right)^{2}
$$

Nielsen (1999) recomienda calcular la resistencia efectiva a tensión del concreto $\left(f_{t e f}\right)$, afectando la resistencia índice $\left(f_{t}\right)$ con varios factores de eficiencia $(v)$. De ellos, sólo se tomó en cuenta el factor $v=0.6$, pues es análogo al que se usa en la construcción del bloque equivalente a compresión del reglamento ACI-318. La resistencia a tensión del concreto se supuso igual a la que se obtiene en la prueba brasileña. De acuerdo con el reglamento ACI-318, ésta es $f_{t}=1.78 \sqrt{f_{c}^{\prime}}$. Al tomar en cuenta tanto el factor de eficiencia $v$ como $f_{t}$, y substituyendo en la ecuación 17, se obtiene alternamente la ecuación 18:

$$
M_{a g r}=0.53 \sqrt{f_{c}^{\prime} b} b\left(\frac{d_{c r}}{\operatorname{sen} \theta}\right)^{2}
$$

En Archundia y Tena (2015a) se demuestra también que, de manera práctica, $V_{c}$ se puede calcular conforme a la ecuación 19 (derivada para $\theta_{E}=45^{\circ}$ ), cuando el peralte y el momento flexionante crecen en la misma dirección, que es el caso en estudio:

$$
V_{c}=0.53 \sqrt{f_{c}^{\prime}} b d_{\min }[1+2.5 \tan \alpha]
$$


A-priori, se sabe que el modelo desarrollado con la teoría de puntales y tensores es conservador en esencia. En general, el método de puntales y tensores se fundamenta en el teorema del límite inferior de la teoría de la plasticidad. Asimismo, el método privilegia la resistencia del acero de refuerzo transversal, y en él se considera la contribución del concreto (agrietamiento diagonal) como una resistencia complementaria válida sólo en trabes esbeltas (Archundia y Tena 2015b). Por ello, en la estimación de la resistencia a cortante involucra el momento flexionante al agrietamiento diagonal $\left(M_{a g r}\right)$, en lugar del momento flexionante desarrollado al cortante máximo $\left(M_{c r}\right)$, como se consideró en el método de la sección crítica. Sin embargo, por congruencia con esta teoría se decide evaluar al modelo utilizando las propuestas para calcular $M_{a g r}$ con esta filosofía. Eso sí, como en todo método para el diseño por cortante, la propuesta del ángulo de la grieta principal por cortante con respecto al plano horizontal $(\theta)$ es fundamental (Archundia y Tena 2015a), y es por ello que se evalúan las dos propuestas para $\theta$ hechas con base en datos experimentales (ecuaciones 6 y 7). Finalmente, cabe señalar que las trabes evaluadas son esbeltas, es decir, tienen al menos una región B perfectamente definida según la recomendación de Archundia y Tena (2015a).

Así, y dada la información experimental disponible, se evaluaron las aproximaciones obtenidas con el modelo de puntales y tensores estudiando las siguientes opciones. Se evaluaron dos opciones para determinar el momento de agrietamiento $\left(M_{a g r}\right)$ : a) considerando que, para fines prácticos, el esfuerzo de tensión efectiva se puede tomar como el esfuerzo de ruptura reportado en la tabla $3\left(f_{t e} \approx f_{r}\right) \mathrm{y}$, por tanto, se emplea la ecuación $17 \mathrm{y}, \mathrm{b}$ ) utilizando la aproximación de la ecuación 18 en función de $f_{c}^{\prime}$ experimental. Con base en ello, se tuvieron entonces dos opciones para evaluar $V_{a g r}$ (ecuación 16) y, por tanto, dos valores para $V_{c}$ (ecuación 14), que sumada a la ecuación aproximada (ecuación 19), dan tres posibles estimaciones del cortante nominal resistente para cada valor del ángulo principal de la grieta considerado. Por lo tanto, al igual que para el método de las secciones, se consideraron: a) $\theta=45-\alpha$ y, b) $\theta=39-0.6 \alpha$. Por lo tanto, en total se evaluaron seis aproximaciones del método, en función de la información que se dispone.

Los resultados así obtenidos se presentan en las tablas 8 a 13, donde se comparan con los cortantes últimos efectivos $\mathrm{V}_{\text {uef }}$ determinados experimentalmente y mostrados en las tablas 1 y 2 . Nuevamente, en las tablas 8 a 13 se indican con un asterisco $(*)$ los cocientes $V_{n} / V_{\text {uef }}$ calculados para el espécimen TASCV $3 \alpha 4-R 1 c$ que un cortante $\mathrm{V}_{\text {uef }}$ que no corresponde al último por la falla prematura del vértice derecho de la cartela.

De la información reportada en las tablas 8 a 10, se pueden hacer las siguientes observaciones, en términos generales, cuando se considera $\theta=45-\alpha$ : a) la estimación del cortante nominal de las trabes acarteladas $V_{n}$ es bastante conservadora para momento negativo. De hecho, si se toma el único valor de la trabe TASCV3 $\alpha 4-R 1 c$ que corresponde a la falla (cartela derecha), la estimación es buena, pero conservadora, b) la estimación del cortante nominal de las trabes acarteladas $V_{n}$ es muy buena y generalmente conservadora para momento positivo, c) como se esperaba, la estimación del cortante del agrietamiento $V_{a g r}$ está demasiado por debajo del que aporta el refuerzo inclinado $\left(V_{r i}\right)$ tanto para momento negativo (tabla 1) como positivo (tabla 2), d) en este sentido, se comprueba con datos experimentales que estimar $M_{a g r}$ con las ecuaciones 17 ó 18 hace muy poca diferencia en la determinación de $V_{a g r}$ (tablas 8 y 9) y, por lo tanto, se comprueba que son expresiones razonablemente análogas, e) como también se esperaba, la contribución del acero de refuerzo transversal es conservadora y se subestima notablemente si se compara con respecto a los determinados experimentalmente, sobre todo a momento negativo y, f) se aprecia que la ecuación simplificada para estimar $V_{c}$ (ecuación 19) tiende a ser demasiado conservadora con respecto a las ecuaciones más rigurosas (ecuaciones 15 y 16) a medida que el ángulo de acartelamiento $\alpha$ aumenta, pues en su derivación y en aras de simplificarla lo más posible, se fijó la 
proyección de la grieta (Archundia y Tena 2015a) y, por tanto, se tiende a subestimar $M_{a g r}$ a medida que $\alpha$ aumenta.

Tabla 8. Cortantes nominales estimados con las ecuaciones de diseño de puntales y tensores cuando $M_{c r}$ se estima con la ec. $17, V_{a g r}$ con la ec. $16, V_{c}$ con la ec. 14 y $\theta=45-\alpha$, momentos positivo y negativo

\begin{tabular}{|c|c|c|c|c|c|c|c|c|c|c|c|c|c|c|}
\hline \multirow[t]{2}{*}{ Espécimen } & \multicolumn{7}{|c|}{ Cartela (viga) izquierda } & \multicolumn{7}{|c|}{ Cartela (viga) derecha } \\
\hline & $\begin{array}{l}V_{p c} \\
(t)\end{array}$ & $\begin{array}{c}V_{\text {agr }} \\
(t)\end{array}$ & $\begin{array}{l}V_{c} \\
(t)\end{array}$ & $\begin{array}{l}V_{s} \\
(t)\end{array}$ & $\begin{array}{l}V_{n} \\
(t)\end{array}$ & $\begin{array}{c}\mathbf{V}_{\mathbf{n}} / \mathbf{V}_{\text {uef }} \\
\text { M- }\end{array}$ & $\begin{array}{c}\mathbf{V}_{\mathrm{n}} / \mathbf{V}_{\text {uef }} \\
\mathrm{M}+\end{array}$ & $\begin{array}{l}V_{\text {pc }} \\
(t)\end{array}$ & $\begin{array}{c}V_{\text {agr }} \\
(t)\end{array}$ & $\begin{array}{l}\mathbf{V}_{\mathbf{c}} \\
(\mathbf{t})\end{array}$ & $\begin{array}{l}V_{s} \\
(t)\end{array}$ & $\begin{array}{l}V_{n} \\
(t)\end{array}$ & $\begin{array}{c}\mathbf{V}_{\mathbf{n}} / \mathbf{V}_{\text {uef }} \\
\mathbf{M}-\end{array}$ & $\begin{array}{c}\mathbf{V}_{\mathbf{n}} / \mathbf{V}_{\text {uef }} \\
\mathbf{M}+\end{array}$ \\
\hline TASCV3a0-R1c & 9.06 & 0.00 & 9.06 & 10.43 & 19.49 & 0.655 & 0.899 & 9.34 & 0.00 & 9.34 & 10.43 & 19.77 & 0.638 & 0.912 \\
\hline TASCV3a1-R1c & 7.40 & 1.66 & 9.06 & 10.62 & 19.68 & 0.675 & 0.976 & 7.44 & 1.71 & 9.15 & 10.62 & 19.77 & 0.712 & 1.116 \\
\hline TASCV3a2-R1c & 6.24 & 2.67 & 8.91 & 10.68 & 19.59 & 0.556 & 0.989 & 6.21 & 2.82 & 9.03 & 10.68 & 19.71 & 0.628 & 0.926 \\
\hline TASCV3a3-R1c & 5.11 & 4.17 & 9.27 & 10.62 & 19.90 & 0.647 & 0.915 & 5.19 & 3.85 & 9.04 & 10.62 & 19.66 & 0.645 & 0.896 \\
\hline TASCV3a4-R1c & 4.88 & 4.99 & 9.87 & 10.99 & 20.86 & $0.976^{*}$ & $1.169^{*}$ & 4.85 & 4.63 & 9.48 & 10.99 & 20.47 & 0.792 & $1.055^{*}$ \\
\hline
\end{tabular}

Tabla 9. Cortantes nominales estimados con las ecuaciones de diseño de puntales y tensores cuando $M_{c r}$ se estima con la ec. $18, V_{a g r}$ con la ec. $16, V_{c}$ con la ec. 14 y $\theta=45-\alpha$, momentos positivo y negativo

\begin{tabular}{|c|c|c|c|c|c|c|c|c|c|c|c|c|c|c|}
\hline \multirow[t]{2}{*}{ Espécimen } & \multicolumn{7}{|c|}{ Cartela (viga) izquierda } & \multicolumn{7}{|c|}{ Cartela (viga) derecha } \\
\hline & $\begin{array}{l}V_{p c} \\
(t)\end{array}$ & $\begin{array}{c}V_{\text {agr }} \\
(t)\end{array}$ & $\begin{array}{l}V_{c} \\
(t)\end{array}$ & $\begin{array}{l}V_{s} \\
(t)\end{array}$ & $\begin{array}{l}V_{n} \\
(t)\end{array}$ & $\begin{array}{c}\mathbf{V}_{\mathbf{n}} / \mathbf{V}_{\text {uef }} \\
M-\end{array}$ & $\begin{array}{c}\mathbf{V}_{\mathbf{n}} / \mathbf{V}_{\text {uef }} \\
\mathbf{M}+\end{array}$ & $\begin{array}{l}V_{p c} \\
(t)\end{array}$ & $\begin{array}{l}V_{\text {agr }} \\
\text { (t) }\end{array}$ & $\begin{array}{l}V_{c} \\
(t)\end{array}$ & $\begin{array}{c}V_{s} \\
(t)\end{array}$ & $\begin{array}{l}V_{n} \\
(t)\end{array}$ & $\begin{array}{c}\mathbf{V}_{\mathrm{n}} / \mathbf{V}_{\text {uef }} \\
\mathbf{M}-\end{array}$ & $\begin{array}{c}\mathbf{V}_{\mathrm{n}} / \mathbf{V}_{\text {uef }} \\
\mathbf{M}+\end{array}$ \\
\hline TASCV3a0-R1c & 9.06 & 0.00 & 9.06 & $\overline{10.43}$ & 19.49 & 0.655 & 0.899 & 9.34 & 0.00 & 9.34 & 10.43 & 19.77 & 0.638 & 0.912 \\
\hline TASCV3 $\alpha 1-R 1 c$ & 7.40 & 1.03 & 8.43 & 10.62 & 19.05 & 0.654 & 0.945 & 7.44 & 1.04 & 8.47 & 10.62 & 19.09 & 0.687 & 1.078 \\
\hline TASCV3a2-R1c & 6.24 & 2.27 & 8.51 & 10.68 & 19.19 & 0.545 & 0.969 & 6.21 & 2.26 & 8.47 & 10.68 & 19.15 & 0.610 & 0.899 \\
\hline TASCV3 $\alpha 3-R 1 c$ & 5.11 & 2.86 & 7.97 & 10.62 & 18.59 & 0.605 & 0.855 & 5.19 & 2.91 & 8.10 & 10.62 & 18.72 & 0.614 & 0.854 \\
\hline TASCV3a4-R1c & 4.88 & 4.59 & 9.47 & 10.99 & 20.46 & $0.957 *$ & $1.147 *$ & 4.85 & 4.56 & 9.41 & 10.99 & 20.40 & 0.789 & $1.051 *$ \\
\hline
\end{tabular}

Tabla 10. Cortantes nominales estimados con las ecuaciones de diseño de puntales y tensores cuando $V_{c}$ se estima con la ec. 19 y $\theta=45-\alpha$, momentos positivo y negativo

\begin{tabular}{|c|c|c|c|c|c|c|c|c|c|c|}
\hline \multirow[t]{2}{*}{ Espécimen } & \multicolumn{5}{|c|}{ Cartela (viga) izquierda } & \multicolumn{5}{|c|}{ Cartela (viga) derecha } \\
\hline & $\begin{array}{l}V_{c} \\
(t)\end{array}$ & $\begin{array}{l}\mathbf{V}_{\mathrm{s}} \\
(\mathbf{t})\end{array}$ & $\begin{array}{l}V_{n} \\
(t)\end{array}$ & $\begin{array}{c}\mathbf{V}_{\mathbf{n}} / \mathbf{V}_{\text {uef }} \\
\text { M- }\end{array}$ & $\begin{array}{c}\mathbf{V}_{\mathbf{n}} / \mathbf{V}_{\text {uef }} \\
\mathbf{M +}\end{array}$ & $\begin{array}{l}V_{c} \\
(t)\end{array}$ & $\begin{array}{l}\mathbf{V}_{\mathbf{s}} \\
(\mathbf{t})\end{array}$ & $\begin{array}{l}V_{n} \\
(t)\end{array}$ & $\begin{array}{c}\mathbf{V}_{\mathbf{n}} / \mathbf{V}_{\text {uef }} \\
\mathbf{M} \text { - }\end{array}$ & $\begin{array}{c}\mathbf{V}_{\mathbf{n}} / \mathbf{V}_{\text {uef }} \\
\mathbf{M}+\end{array}$ \\
\hline TASCV3a0-R1c & 9.06 & 10.43 & 19.49 & 0.655 & 0.899 & 9.34 & 10.43 & 19.77 & 0.638 & 0.912 \\
\hline TASCV3a2-R1c & 7.46 & 10.68 & 18.15 & 0.515 & 0.917 & 7.43 & 10.68 & 18.11 & 0.577 & 0.851 \\
\hline TASCV3a3-R1c & 6.34 & 10.62 & 16.96 & 0.552 & 0.780 & 6.44 & 10.62 & 17.06 & 0.560 & 0.778 \\
\hline TASCV3a4-R1c & 6.29 & 10.99 & 17.28 & $0.809 *$ & $0.969 *$ & 6.25 & 10.99 & 17.24 & 0.667 & $0.888^{*}$ \\
\hline
\end{tabular}

En las tablas 11 a 13 se presentan los resultados cuando se emplea un ángulo de la grieta principal por cortante $\theta=39-0.6 \alpha$, más cercano a la regresión de todos los datos experimentales confiables disponibles (figura 14). Se confirma que utilizando esta propuesta para $\theta$ se mejoran las aproximaciones para momento negativo. En contraste, y como se esperaba, para momento positivo la aproximación en unos casos mejora, pero en general disminuye, pues empiezan a dominar las sobrestimaciones.

Como en el diseño por sismo más carga vertical de una viga continua va a dominar la condición del momento negativo sobre positivo para definir el refuerzo por cortante de la viga en sus extremos, se puede concluir entonces que el método de puntales y tensores es una opción confiablemente conservadora si existe una propuesta razonable del ángulo de la grieta principal por cortante, $\theta$. Es por ello que se reitera que es necesario compilar una base de datos experimentales más grande y extensa que permita definir una ecuación del tipo $\theta=\theta_{0}-a_{1} \alpha$, donde $\theta_{0}$ es el ángulo medio estadístico de la grieta principal por cortante para vigas prismáticas y $a_{l}$ es la pendiente media estadística de los datos experimentales de esa grieta obtenidos en trabes acarteladas. 
Tabla 11. Cortantes nominales estimados con las ecuaciones de diseño de puntales y tensores cuando $M_{c r}$ se estima con la ec. $17, V_{a g r}$ con la ec. $16, V_{c}$ con la ec. 14 y $\theta=39-0.6 \alpha$, momentos positivo y negativo

\begin{tabular}{|c|c|c|c|c|c|c|c|c|c|c|c|c|c|c|}
\hline \multirow{2}{*}{ Espécimen } & \multicolumn{7}{|c|}{ Cartela (viga) izquierda } & \multicolumn{7}{|c|}{ Cartela (viga) derecha } \\
\hline & $\begin{array}{l}V_{p c} \\
(t)\end{array}$ & $\begin{array}{c}V_{\text {agr }} \\
(t)\end{array}$ & $\begin{array}{l}V_{c} \\
(t)\end{array}$ & $\begin{array}{l}V_{s} \\
(t)\end{array}$ & $\begin{array}{l}V_{n} \\
(t)\end{array}$ & $\begin{array}{c}\mathbf{V}_{\mathbf{n}} / \mathbf{V}_{\text {uef }} \\
\text { M- }\end{array}$ & $\begin{array}{c}\mathbf{V}_{\mathrm{n}} / \mathbf{V}_{\text {uef }} \\
\mathrm{M}+\end{array}$ & $\begin{array}{l}V_{p c} \\
(t)\end{array}$ & $\begin{array}{l}V_{\text {agr }} \\
\text { (t) }\end{array}$ & $\begin{array}{l}V_{c} \\
(t)\end{array}$ & $\begin{array}{l}\mathbf{V}_{\mathrm{s}} \\
(\mathrm{t})\end{array}$ & $\begin{array}{l}V_{n} \\
(t)\end{array}$ & $\begin{array}{c}\mathbf{V}_{\mathrm{n}} / \mathbf{V}_{\text {uef }} \\
\mathrm{M}-\end{array}$ & $\begin{array}{c}\mathbf{V}_{\mathbf{n}} / \mathbf{V}_{\text {uef }} \\
\mathbf{M}+\end{array}$ \\
\hline TASCV3a0-R1c & 9.06 & 0.00 & 9.06 & 12.88 & 21.94 & 0.737 & 1.027 & 9.34 & 0.00 & 9.34 & 12.88 & 22.22 & 0.717 & 1.025 \\
\hline TASCV3a1-R1C & 7.44 & 2.08 & 9.52 & 12.80 & 22.32 & 0.766 & 1.107 & 7.48 & 2.14 & 9.62 & 12.80 & 22.42 & 0.807 & 1.266 \\
\hline TASCV3a2-R1c & 6.29 & 3.27 & 9.57 & 12.53 & 22.09 & 0.627 & 1.116 & 6.27 & 3.46 & 9.73 & 12.53 & 22.25 & 0.709 & 1.045 \\
\hline TASCV3a3-R1c & 5.15 & 5.01 & 10.17 & 12.23 & 22.39 & 0.727 & 1.029 & 5.24 & 4.63 & 9.87 & 12.23 & 22.09 & 0.724 & 1.007 \\
\hline TASCV3a4-R1c & 4.92 & 5.79 & 10.71 & 12.28 & 22.99 & $1.076^{*}$ & $1.289 *$ & 4.89 & 5.37 & 10.26 & 12.28 & 22.54 & 0.872 & $1.161 *$ \\
\hline
\end{tabular}

Tabla 12. Cortantes nominales estimados con las ecuaciones de diseño de puntales y tensores cuando $M_{c r}$ se estima con la ec. $18, V_{a g r}$ con la ec. $16, V_{c}$ con la ec. 14 y $\theta=39-0.6 \alpha$, momentos positivo y negativo

\begin{tabular}{|c|c|c|c|c|c|c|c|c|c|c|c|c|c|c|}
\hline \multirow[t]{2}{*}{ Espécimen } & \multicolumn{7}{|c|}{ Cartela (viga) izquierda } & \multicolumn{7}{|c|}{ Cartela (viga) derecha } \\
\hline & $\begin{array}{l}\mathbf{V}_{\mathrm{pc}} \\
(\mathbf{t})\end{array}$ & $\begin{array}{c}V_{\text {agr }} \\
(t)\end{array}$ & $\begin{array}{l}V_{c} \\
(t)\end{array}$ & $\begin{array}{l}V_{s} \\
(t)\end{array}$ & $\begin{array}{l}V_{n} \\
(t)\end{array}$ & $\begin{array}{c}\mathbf{V}_{\mathbf{n}} / \mathbf{V}_{\text {uef }} \\
\text { M- }\end{array}$ & $\begin{array}{c}\mathbf{V}_{\mathbf{n}} / \mathbf{V}_{\text {uef }} \\
\mathrm{M}+\end{array}$ & $\begin{array}{l}V_{p c} \\
(t)\end{array}$ & $\begin{array}{c}V_{\text {agr }} \\
(t)\end{array}$ & $\begin{array}{l}V_{c} \\
(t)\end{array}$ & $\begin{array}{l}\mathbf{V}_{\mathrm{s}} \\
(\mathrm{t})\end{array}$ & $\begin{array}{l}V_{n} \\
(t)\end{array}$ & $\begin{array}{c}\mathbf{V}_{\mathbf{n}} / \mathbf{V}_{\text {uef }} \\
\mathbf{M}-\end{array}$ & $\begin{array}{c}\mathbf{V}_{\mathbf{n}} / \mathbf{V}_{\text {uef }} \\
\mathbf{M}+\end{array}$ \\
\hline TASCV3a0-R1c & 9.06 & 0.00 & 9.06 & 12.88 & 21.94 & 0.737 & 1.027 & 9.34 & 0.00 & 9.34 & 12.88 & 22.22 & 0.717 & 1.025 \\
\hline TASCV3a1-R1c & 7.44 & 1.29 & 8.73 & 12.80 & 21.53 & 0.739 & 1.068 & 7.48 & 1.30 & 8.78 & 12.80 & 21.58 & 0.777 & 1.219 \\
\hline TASCV3a2-R1c & 6.29 & 2.78 & 9.08 & 12.53 & 21.60 & 0.613 & 1.091 & 6.27 & 2.77 & 9.04 & 12.53 & 21.56 & 0.687 & 1.013 \\
\hline TASCV3a3-R1c & 5.15 & 3.44 & 8.60 & 12.23 & 20.82 & 0.677 & 0.957 & 5.24 & 3.50 & 8.74 & 12.23 & 20.96 & 0.687 & 0.956 \\
\hline TASCV3a4-R1c & 4.92 & 5.32 & 10.25 & 12.28 & 22.53 & $1.054 *$ & $1.263 *$ & 4.89 & 5.29 & 10.18 & 12.28 & 22.46 & 0.869 & $1.157^{*}$ \\
\hline
\end{tabular}

Tabla 13. Cortantes nominales estimados con las ecuaciones de diseño de puntales y tensores cuando $V_{c}$ se estima con la ec. 19 y $\theta=39-0.6 \alpha$, momentos positivo y negativo

\begin{tabular}{|c|c|c|c|c|c|c|c|c|c|c|}
\hline \multirow[t]{2}{*}{ Espécimen } & \multicolumn{5}{|c|}{ Cartela (viga) izquierda } & \multicolumn{5}{|c|}{ Cartela (viga) derecha } \\
\hline & $\begin{array}{l}V_{c} \\
(t)\end{array}$ & $\begin{array}{l}V_{s} \\
(t)\end{array}$ & $\begin{array}{l}V_{n} \\
(t)\end{array}$ & $\begin{array}{c}\mathbf{V}_{\mathbf{n}} / \mathbf{V}_{\text {uef }} \\
\text { M- }\end{array}$ & $\begin{array}{c}\mathbf{V}_{\mathbf{n}} / \mathbf{V}_{\text {uef }} \\
\mathbf{M}_{+}\end{array}$ & $\begin{array}{l}V_{c} \\
(t)\end{array}$ & $\begin{array}{l}V_{s} \\
(t)\end{array}$ & $\begin{array}{l}V_{n} \\
(t)\end{array}$ & $\begin{array}{c}\mathbf{V}_{\mathbf{n}} / \mathbf{V}_{\text {uef }} \\
\mathrm{M} \text { - }\end{array}$ & $\begin{array}{c}\mathbf{V}_{\mathbf{n}} / \mathbf{V}_{\text {uef }} \\
\mathbf{M +}\end{array}$ \\
\hline TASCV3a0-R1c & 9.06 & 12.88 & 21.94 & 0.737 & 1.027 & 9.34 & 12.88 & 22.22 & 0.717 & 1.025 \\
\hline TASCV3a2-R1c & 7.46 & 12.53 & 19.99 & 0.567 & 1.010 & 7.43 & 12.53 & 19.96 & 0.636 & 0.938 \\
\hline TASCV3a3-R1c & 6.34 & 12.23 & 18.56 & 0.604 & 0.853 & 6.44 & 12.23 & 18.66 & 0.612 & 0.851 \\
\hline TASCV3a4-R1c & 6.29 & 12.28 & 18.57 & $0.869 *$ & $1.041^{*}$ & 6.25 & 12.28 & 18.53 & 0.717 & $0.955^{*}$ \\
\hline
\end{tabular}

\section{COMENTARIOS FINALES}

Se presentaron los resultados e interpretaciones de los ensayes de trabes acarteladas de concreto reforzado en condiciones de continuidad y sujetas a cargas cíclicas reversibles que permita estudiar su mecanismo resistente a cortante. Todos los especímenes tienen una longitud de acartelamiento de un tercio del claro libre y cubren uniformemente ángulos de acartelamiento entre cero (prismática) y diez grados. Las variables en estudio con respecto al mecanismo resistente a cortante fueron: (a) el ángulo de acartelamiento y su impacto en la resistencia del concreto, (b) la contribución del acero de refuerzo longitudinal inclinado, (c) la contribución del refuerzo transversal y d) el ángulo de la grieta principal por cortante.

Con base en el procesado de los resultados capturados de la instrumentación externa e interna de los experimentos, se pueden hacer las siguientes observaciones con respecto al mecanismo resistente a cortante:

- A partir de la ubicación del eje neutro a lo largo del eje longitudinal de las cartelas con base en las deformaciones medidas en el acero de refuerzo longitudinal superior e inferior, se comprueba que aún a la carga última y con un patrón de agrietamiento importante, se sigue 
presentando un acoplamiento importante a flexión y carga axial en las trabes acarteladas que lidera a lo que se conoce como "mecanismo de arco" ("arching action"), particularmente cuando el refuerzo longitudinal inclinado trabaja a compresión (momento negativo), lo cual coincide plenamente con lo reportado recientemente por Hou et al. (2015) en sus ensayes ante carga estática monótona creciente.

- Antes del primer agrietamiento diagonal, la contribución al cortante del acero de refuerzo transversal es prácticamente despreciable, pero se incrementa rápidamente a partir de ahí y resiste la mayor parte del cortante nominal, tanto a momento positivo como negativo.

- Se observó que la resistencia del concreto se degrada a distorsiones menores en las trabes prismáticas con respecto a las trabes acarteladas.

- Con respecto a la contribución del refuerzo inclinado a la resistencia al corte de trabes acarteladas, se apreció que su contribución es evidente aún antes del primer agrietamiento diagonal. Como se esperaba, su contribución aumenta a medida que el ángulo de acartelamiento aumenta. Se apreció que, a medida que aumenta el ángulo de acartelamiento $\alpha$, éste se convierte en el mecanismo resistente principal del concreto a cortante, aún a distorsiones pequeñas. De hecho, se observó que el refuerzo inclinado prácticamente toma toda la resistencia a cortante del concreto para distorsiones mayores a $1 \%$. Por ello, el cortante que aporta el refuerzo inclinado en trabes acarteladas es el único que se suma a la contribución de los estribos en las cargas máximas y de colapso, pues el concreto en sí agotó prácticamente su resistencia.

- Las trabes acarteladas resisten un cortante último efectivo similar o aún mayor que las trabes prismáticas de referencia, sobre todo a medida que aumenta el ángulo de acartelamiento y particularmente a momento negativo. La resistencia a cortante adicional de las trabes acarteladas a un menor volumen de concreto se debe a dos razones: 1) un mayor aporte del refuerzo longitudinal inclinado, tanto a momento positivo y negativo, la cual en general se incrementa a medida que aumenta el ángulo de acartelamiento $\alpha \mathrm{y}, 2$ ) en general, el refuerzo transversal por cortante participa más en la resistencia a medida que aumenta el ángulo de acartelamiento $\alpha$, como consecuencia de que el ángulo de inclinación de la grieta principal por cortante $\theta$ disminuye.

Con la finalidad de mejorar las ecuaciones de diseño propuestas en trabajos anteriores, se actualizaron con base experimental las ecuaciones para estimar: a) los momentos flexionantes efectivamente desarrollados a la carga máxima o última, $V_{u}$ en las secciones críticas por cortante de las trabes acarteladas y, b) el ángulo de la grieta principal por cortante, $\theta$. Con respecto a esto último, cabe destacar que se observó que, en términos generales, los ángulos de inclinación de la grieta por cortante son mayores en condiciones de apoyo simple que en condición de continuidad. Por ello, al parecer, la contribución de los estribos a la resistencia a cortante es, en general, más importante en una viga continua que en una viga simplemente apoyada. Esto parece cumplirse también para las vigas prismáticas. Dado que las ecuaciones de diseño disponibles en la mayoría de los reglamentos de concreto reforzado tiene como base los resultados de ensayes de vigas prismáticas simplemente apoyadas, se intuye que, para fines prácticos, el diseño por cortante de vigas continuas pudiera ser conservador empleando esas ecuaciones. Como se esperaba, también se comprobó que, generalmente, el ángulo de inclinación de la grieta principal a cortante es mayor para trabes sin refuerzo por cortante que para trabes con refuerzo por cortante. Se observó también que el ángulo de inclinación de la grieta depende del tipo de carga aplicada, resultando en general menor para cargas cíclicas, aunque con una mayor dispersión de los datos obtenidos. 
Con base en la inclusión de estas mejoras actualizadas, se evaluaron ecuaciones de diseño propuestas para el diseño a cortante basadas en: a) el método de las secciones y, b) el método de puntales y tensores (modelos de armadura), que se confrontaron con los resultados determinados experimentalmente. Se comprueba que, para fines prácticos, dichas ecuaciones permite estimar valores de resistencia a cortante razonables para fines de diseño.

En general, con el método de la sección crítica se obtienen estimaciones razonablemente cercanas, pero conservadoras del cortante máximo para momento negativo $\mathrm{y}$, razonables, pero no conservadoras, para momento positivo.

Con el método de puntales y tensores se obtienen estimaciones conservadoras del cortante máximo para momento negativo, como una consecuencia lógica que, en esencia, el método es conservador, pues en la estimación de la resistencia a cortante involucra el momento flexionante al agrietamiento diagonal $\left(M_{a g r}\right)$, en lugar del momento flexionante desarrollado al cortante máximo $\left(M_{c r}\right)$, como se consideró en el método de la sección crítica. En cambio, las estimaciones para el cortante máximo a momento positivo son muy cercanas.

Como en el diseño por sismo más carga vertical de una viga continua va a dominar la condición del momento negativo sobre el momento positivo para definir el refuerzo por cortante de la viga en sus extremos, se puede concluir entonces que el método de las secciones que se propone es conservador, pero razonablemente y que el método de puntales y tensores es también una opción confiable, pero más conservadora. En ambos métodos es fundamental que exista una propuesta razonable del ángulo de la grieta principal por cortante, $\theta$. Es por ello que se recomienda en estudios futuros, como muy necesario, compilar una base de datos experimentales más grande y extensa que permita definir una ecuación del tipo $\theta=\theta_{0}-a_{1} \alpha$, donde $\theta_{0}$ es el ángulo medio estadístico de la grieta principal por cortante para vigas prismáticas y $a_{l}$ es la pendiente media estadística de los datos experimentales de esa grieta obtenidos en trabes acarteladas. Mientras tanto, las ecuaciones propuestas por los autores para $\theta$ con base experimental son bastante razonables y pueden usarse con confianza.

\section{AGRADECIMIENTOS}

Este trabajo formó parte del proyecto 79878 de Ciencia Básica de Conacyt intitulado Comportamiento cíclico a flexión y cortante de trabes acarteladas de concreto reforzado continuas, cuyo apoyo agradecen encarecidamente los autores. Agradecemos a la empresa HESA, en particular al Ing. José Antonio Sánchez Miravete, por asistirnos en la fabricación del acero de refuerzo (longitudinal y estribos), particularmente el inclinado. Durante la elaboración de los trabajos experimentales, intervinieron un gran número de personas, por lo que los autores agradecen a las siguientes personas de la UAM-A. Al maestro del laboratorio Rubén Barrera, Técnico José Luis Caballero, Técnico Juan Mateos, M. en I. Gilberto Rangel Torres y Sra. Silvia Ledesma por su apoyo institucional en el laboratorio. Al ingeniero Edznab López Rosas, quien participó y apoyó en estos experimentos como parte de su tesis de licenciatura y sus seminarios de tesis de maestría. Al ingeniero Jesús Iram Aranda Carrillo, que ensayó el espécimen TASCV $3 \alpha 4-R 1 c$ como parte de sus proyectos terminales y apoyó los experimentos como parte de sus talleres de ingeniería civil. El Ing. Eduardo Antonio Ayala Espejel apoyó a los experimentos como parte de su proyecto de integración y de sus talleres de ingeniería civil. El Ing. Luis Antonio Mendoza Valdez apoyó estos experimentos como parte de su proyecto terminal de licenciatura. El Ing. José Daniel Rivera Castro apoyó a los experimentos dentro de sus labores de ayudantía en el Área de Estructuras. Los ingenieros Ricardo Flores, Gadiel Martínez, Aldo Mendoza, Enrique Huerta, Juan Carlos Rodríguez, Mario Medina, Jovani Martínez, Sergio Reyes y Armando Colín, colaboraron tanto en sus talleres de 
ingeniería civil como en su servicio social. A todos los alumnos y alumnas que participaron en este proyecto como parte de sus talleres de ingeniería civil: Andrés Gama, Angel Liga, Alejandro Pérez, Gloria Saldívar, Nelly Orozco, Paola Martínez, Saraí Cisneros, Miriam Hernández, Carolina Viatela, Erik González, Irving Chávez, Erik Sánchez, Francisco Juárez, Fernando Arriaga, José Roldán, Juan Flores, Juan Sánchez, Julio Castaños, Oliver Narcizo, Saúl Rico, Carlos Morales, Jovani Cathi, Manuel López, Alex Cabrera, Abigail Velasco, Emmanuel Martínez, Jonathan Zendejas, Isaac Marín, Alan Herrera, Alan Hernández, Marco Islas, Jorge Rodríguez, Edgar Rojas, Erick Esquivel y Juan Antaño.

\section{REFERENCIAS}

ACI-318-14 (2014), "Building code requirements for structural concrete (ACI-318-14) and commentary (ACI 318R-14)", American Concrete Institute, Farmington Hills, Michigan, USA.

Aranda, J. I. (2015), "Estudio experimental del cortante en vigas acarteladas de concreto reforzado, con ángulos de $8^{\circ}$ y $10^{\circ}$ en cartela, construcción, instrumentación y demolición", Proyectos Terminales I y II, Departamento de Materiales, Universidad Autónoma Metropolitana, septiembre.

Archundia, H. I. (2004), "Comportamiento a cortante de trabes acarteladas de concreto reforzado ante carga estática", Tesis de Maestría, División de Estudios de Posgrado de la Facultad de Ingeniería, Universidad Nacional Autónoma de México, mayo.

Archundia, H. I., A. Tena y O. M. González (2005), "Estudio experimental del cortante estático en trabes acarteladas de concreto reforzado", Reporte de Investigación 453, División de Ciencias Básicas e Ingeniería, Universidad Autónoma Metropolitana, junio, ISBN 970-31-0491-6.

Archundia, H. I., A. Tena y O. M. González (2006), "Mecanismos de resistencia y deformación a cortante de trabes acarteladas de concreto reforzado", Revista Internacional de Ingeniería de Estructuras, Vol. 11, No. 1, pp. 1-23.

Archundia, H. I., A. Tena, A. Grande y O. M. González (2007), "Comportamiento cíclico de trabes acarteladas de concreto reforzado sin refuerzo transversal que fallan por cortante", Revista de Ingeniería Sísmica, No. 76, pp. 89-112, enero-junio. DOI: https://doi.org/10.18867/ris-0.

Archundia-Aranda, H. I. y A. Tena-Colunga (2008), "Cyclic behavior of reinforced concrete haunched beams failing in shear", Memorias, $14^{\text {th }}$ World Conference on Earthquake Engineering, Beijing, China, Artículo No. 12-01-0105, CD-ROM, octubre.

Archundia-Aranda, H. I., A. Tena-Colunga y A. Grande-Vega (2013), "Behavior of reinforced concrete haunched beams subjected to cyclic shear", Engineering Structures, Vol. 49, pp. 27-42, http://dx.doi.org/10.1016/j.engstruct.2012.10.037.

Archundia, H. I. (2013), "Recomendaciones de diseño a cortante de trabes acarteladas de concreto reforzado", Tesis de Doctorado, División de Estudios de Posgrado de la Facultad de Ingeniería, Universidad Nacional Autónoma de México, diciembre.

Archundia, H. I. y A. Tena (2015a), "Diseño racional a cortante de trabes acarteladas de concreto reforzado", Concreto y Cemento. Investigación y Desarrollo, Vol. 6, No. 2, pp. 2-29, junio.

Archundia, H. I. y A. Tena (2015b), "Sección critica, esbeltez y regiones B-D para diseño a cortante de trabes de concreto reforzado", Concreto y Cemento. Investigación y Desarrollo, Vol. 7, No. 1, pp. 2-29, diciembre.

Debaiky, S.Y. y E. I. El-Niema (1982), "Behavior and strength of reinforced concrete haunched beams in shear”, ACI Journal, Vol. 79, No.3, pp. 184-194. 
El-Mezaini, N., C. Balkaya y E. Çitipitioglu (1991), "Analysis of frames with nonprismatic members", ASCE Journal of Structural Engineering, Vol. 117, No.6, pp. 1573-1592. https://doi.org/10.1061/(ASCE)0733-9445(1991)117:6(1573).

El-Niema, E.I. (1988), "Investigation of concrete haunched beams under shear", ASCE Journal of Structural Engineering, Vol. 114, No. 4, pp. 917-930. https://doi.org/10.1061/(ASCE)07339445(1988)114:4(917).

Grande, A. (2005), "Comportamiento a cortante de trabes acarteladas de concreto reforzado sujetas a cargas cíclicas", Proyectos Terminales I y II, Departamento de Materiales, Universidad Autónoma Metropolitana, julio.

Grande, A. (2009), "Mecanismos de resistencia y deformación a cortante de trabes acarteladas de concreto reforzado sujetas a cargas cíclicas", Tesis de Maestría, Posgrado en Ingeniería Estructural, División de Ciencias Básicas e Ingeniería, Universidad Autónoma Metropolitana Azcapotzalco, julio.

Hou, C., K. Matsumoto y J. Niwa (2015), "Shear failure mechanism of reinforced concrete haunched beams", Journal of Japan Society of Civil Engineers, Vol. 3, No. 1, pp. 230-245. http://doi.org/10.2208/journalofjsce.3.1_230.

MacLeod, I. A. y A. Houmsi (1994), "Shear strength of haunched beams without shear reinforcement", ACI Structural Journal, Vol. 91, No.1, pp. 79-89.

Mörsch, E. (1952), Teoría y práctica del hormigón armado, Tomo II, Gili, Argentina.

Nielsen, M. P. (1999), Limit analysis and concrete plasticity, segunda edición, CRC Press, E.U.A.

Park R. y T. Paulay (1997), Estructuras de concreto reforzado, novena reimpresión de la primera edición, Limusa, México.

Pérez-Caldentey, A., P. Padilla, A. Muttoni y M. Fernández-Ruiz (2012), "Effect of load distribution and variable depth on shear resistance of slender beams without stirrups", ACI Structural Journal, Vol. 109, No. 5, pp. 595-604.

Stefanou, G. D. (1983), "Shear resistance for reinforced concrete beams with non-prismatic sections", Engineering Fracture Mechanics, Vol. 18, No. 3, pp. 643-667. https://doi.org/10.1016/00137944(83)90057-7.

Tena-Colunga, A., H. I. Archundia-Aranda, A. Grande-Vega y O. M. González-Cuevas (2007), "Cyclic shear behavior of reinforced concrete haunched beams", Memorias, Ninth Canadian Conference on Earthquake Engineering (9CCEE), Ottawa, Canadá, CD-ROM, junio.

Tena-Colunga, A., H. I. Archundia-Aranda y O. M. González-Cuevas (2008), "Behavior of reinforced concrete haunched beams subjected to static shear loading", Engineering Structures, Vol. 30, No. 2, pp. 478-492. doi:10.1016/j.engstruct.2007.04.017.

Tena, A., L. A. Urbina, H. I. Archundia y E. López (2014), “Comportamiento cíclico a cortante de trabes acarteladas de concreto reforzado continuas", Memorias, XIX Congreso Nacional de Ingeniería Estructural, Puerto Vallarta, Jalisco, CDROM, Artículo 10-13, pp. 1-28, noviembre.

Tena, A., L. A. Urbina y H. I. Archundia (2017), "Trabes acarteladas de concreto reforzado continuas diseñadas para fallar por cortante. Parte 1: Descripción de los experimentos y del comportamiento cíclico”, Revista de Ingeniería Sísmica, No. 97.

Urbina, L. A. (2013), "Comportamiento a cortante de trabes acarteladas continuas de concreto reforzado sujetas a cargas cíclicas", Tesis de Maestría, Posgrado en Ingeniería Estructural, División de Ciencias Básicas e Ingeniería, Universidad Autónoma Metropolitana Azcapotzalco, diciembre. 